

\title{
Inverse problems for boundary triples with applications
}

\author{
by
}

\author{
B. M. Brown (Cardiff), M. Marletta (Cardiff),
} S. Naboko (St. Petersburg) and I. Wood (Canterbury)

\begin{abstract}
This paper discusses the inverse problem of how much information on an operator can be determined/detected from 'measurements on the boundary'. Our focus is on non-selfadjoint operators and their detectable subspaces (these determine the part of the operator 'visible' from 'boundary measurements').

We show results in an abstract setting, where we consider the relation between the $M$ function (the abstract Dirichlet to Neumann map or the transfer matrix in system theory) and the resolvent bordered by projections onto the detectable subspaces. More specifically, we investigate questions of unique determination, reconstruction, analytic continuation and jumps across the essential spectrum.

The abstract results are illustrated by examples of Schrödinger operators, matrixdifferential operators and, mostly, by multiplication operators perturbed by integral operators (the Friedrichs model), where we use a result of Widom to show that the detectable subspace can be characterized in terms of an eigenspace of a Hankel-like operator.
\end{abstract}

1. Introduction. In this paper we consider inverse problems in a boundary triple setting involving a formally adjoint pair of operators $A$ and $\widetilde{A}$ [35]. We define, and develop formulae for, the detectable subspaces associated with the information available from the abstract Dirichlet to Neumann maps or Titchmarsh-Weyl functions $M(\lambda)$ (see Definitions 2.2 and 2.7). Our focus is on non-selfadjoint operators, but some of the results are new even in the symmetric case.

In the formally symmetric case V. Derkach and M. Malamud [21] (see also Ryzhov [44]) show that if the reducing subspace corresponding to the simple part of the operator (which is a special case of the detectable subspace) is the whole Hilbert space, then the operator can be reconstructed up to unitary equivalence. In terms of the $Q$-function, this result was proved earlier by

2010 Mathematics Subject Classification: 47A10, 30H10, 34B09, 47A11, 47B35.

Key words and phrases: detectable subspace, inverse problem, $M$-function, Friedrichs model, Widom.

Received 18 May 2016; revised 30 October 2016.

Published online *. 
M. Kreı̆n, H. Langer and B. Textorius [33, 34]. These kinds of results go back to M. Livšic who proved the reconstruction up to unitary equivalence of the simple part of an operator from its characteristic function [14]. If the underlying operator is not symmetric, but the detectable subspace is the whole Hilbert space, then the $M$-function determines the operators of an adjoint pair up to weak equivalence [37]. However, weak equivalence does not preserve the spectral properties of the operators. Improving the result on weak equivalence in some special cases is the topic of $[4-6,28]$.

In the last decade there has been an explosion of research on operators in the setting of boundary triples, in particular around their application to partial differential equations, usually in the selfadjoint case (see e.g. $[1-3,7-12,15,17,21-25,27,28,32,36,41,42,44])$. In the PDE case, the main challenge was to describe concrete choices of boundary mappings. Classical 'trace' and 'normal derivative' do not have the requisite properties; for instance, boundary integrals of the form $\int_{\partial \Omega}\left\{\frac{\partial u}{\partial n} \bar{v}-u \frac{\partial v}{\partial n}\right\}$ cannot generally be separated into a difference of two convergent integrals in the manner suggested by the notation. Two different approaches have been used to overcome this: one involves considering such expressions on sets of sufficiently smooth functions and then taking closures [7], requiring the additional abstract concept of quasi-boundary triple; the other involves replacing the normal derivative by a regularized version thereof [17] and allows standard boundary triples to be used directly. Some interesting ODE applications have also appeared, such as the common eigenvalue problem [40]. Generalizations to relations have been studied e.g. in [20,38].

Ryzhov's study of abstract inverse problems [44] mentions regular elliptic PDEs as an illustration. However there are the fundamental differences between Schrödinger operators in dimension $d=1$ [13] and dimension $d>1$ : for $d>1$, the potential can be uniquely recovered from the knowledge of the Dirichlet to Neumann map at a single value of the spectral parameter $[29,47,48]$. The fact that this is not true for $d=1$ indicates that abstract techniques may require more data than are really necessary in some concrete settings, and must be supplemented by a detailed study of the concrete operators to which they will be applied.

In this paper we examine the extent to which the following questions can be answered at an abstract level and in specific examples.

(1) Is the function $M(\lambda)$ uniquely determined from the knowledge of resolvents reduced to the detectable subspace?

(2) To what extent can the bordered resolvent, i.e. the resolvent bordered by projections onto the detectable subspaces related to $A$ and $\widetilde{A}$, be determined from $M(\lambda)$ ? 
(3) What can be said about the relationship between analytic continuation of $M(\lambda)$ and analytic continuation of the bordered resolvent?

(4) What is the relationship between the rank of the jump in $M(\lambda)$ and the rank of the jump in the bordered resolvent across a line of essential spectrum, without loss of generality the real axis, when one has a limiting absorption principle?

(5) To what extent can the detectable subspace be explicitly described in specific examples?

The paper is arranged as follows. Section 2 introduces concepts that will be needed throughout the paper: boundary triples, $M$-functions, solution operators and the detectable subspace. Section 3 shows the concrete realizations of these abstract objects for Schrödinger operators, Hain-Lüst-type operators and the Friedrichs model. In Section 4, we prove that the $M$-function is uniquely determined by one bordered resolvent. Moreover, it can be reconstructed from one bordered resolvent and two closed solution operator ranges or by two bordered resolvents associated with different boundary conditions. We show that the bordered resolvent can be determined from the $M$-function and a family of solution operator ranges. Section 5 examines simultaneous analytic continuation of the $M$-function and bordered resolvents, significantly extending the results on this in [16]. Section 6 deals with jumps of the $M$-function and the bordered resolvent across the essential spectrum. We show that the multiplicity of the a.c. spectrum of the operator reduced to the detectable subspaces coincides with the rank of the jump of the $M$-function. Section 7 determines the detectable subspace and the relation between the $M$-function and bordered resolvent for different parameters of the Friedrichs model. We consider the Friedrichs model as a key example for the development of the theory of detectable subspaces, because it allows a precise description of the structure of the detectable subspace in many cases, while exhibiting such a variety of behaviours that one can hardly expect to obtain a description of the space in all cases in unique terms. In particular, it shows that the reconstruction of the detectable part of the operator from the $M$-function, well-known for Sturm-Liouville problems [13, 39], is not always possible (see e.g. Remark 7.17).

2. Preliminaries: the detectable subspace. This section introduces concepts and notation that will be used throughout the article, and extends some results from previous papers which are needed later to develop the theory. We make the following assumptions.

(1) $A, \widetilde{A}$ are closed, densely defined operators in a Hilbert space $H$.

(2) $A, \widetilde{A}$ are an adjoint pair, i.e. $A^{*} \supseteq \widetilde{A}$ and $\widetilde{A}^{*} \supseteq A$. 
Proposition 2.1 (Lyantse, Storozh, '83 [35]). For each adjoint pair of closed densely defined operators on $H$, there exist "boundary spaces" $\mathcal{H}, \mathcal{K}$ and "trace operators"

$\Gamma_{1}: D\left(\widetilde{A}^{*}\right) \rightarrow \mathcal{H}, \quad \Gamma_{2}: D\left(\widetilde{A}^{*}\right) \rightarrow \mathcal{K}, \quad \widetilde{\Gamma}_{1}: D\left(A^{*}\right) \rightarrow \mathcal{K}, \quad \widetilde{\Gamma}_{2}: D\left(A^{*}\right) \rightarrow \mathcal{H}$ such that for $u \in D\left(\widetilde{A}^{*}\right)$ and $v \in D\left(A^{*}\right)$ we have an abstract Green formula

$$
\left\langle\widetilde{A}^{*} u, v\right\rangle_{H}-\left\langle u, A^{*} v\right\rangle_{H}=\left\langle\Gamma_{1} u, \widetilde{\Gamma}_{2} v\right\rangle_{\mathcal{H}}-\left\langle\Gamma_{2} u, \widetilde{\Gamma}_{1} v\right\rangle_{\mathcal{K}} .
$$

The trace operators $\Gamma_{1}, \Gamma_{2}, \widetilde{\Gamma}_{1}$ and $\widetilde{\Gamma}_{2}$ are bounded with respect to the graph norm. The pair $\left(\Gamma_{1}, \Gamma_{2}\right)$ is surjective onto $\mathcal{H} \times \mathcal{K}$ and $\left(\widetilde{\Gamma}_{1}, \widetilde{\Gamma}_{2}\right)$ is surjective onto $\mathcal{K} \times \mathcal{H}$. Moreover,

(2.2) $D(A)=D\left(\widetilde{A}^{*}\right) \cap \operatorname{ker} \Gamma_{1} \cap \operatorname{ker} \Gamma_{2}, \quad D(\widetilde{A})=D\left(A^{*}\right) \cap \operatorname{ker} \widetilde{\Gamma}_{1} \cap \operatorname{ker} \widetilde{\Gamma}_{2}$.

The collection $\left\{\mathcal{H} \oplus \mathcal{K},\left(\Gamma_{1}, \Gamma_{2}\right),\left(\widetilde{\Gamma}_{1}, \widetilde{\Gamma}_{2}\right)\right\}$ is called a boundary triple for the adjoint pair $A, \widetilde{A}$.

Malamud and Mogilevskii [38] use this setting to define Weyl $M$-functions associated with boundary triples. In [17], we used a slightly different setting in which the boundary conditions and Weyl function contain an additional operator $B \in \mathcal{L}(\mathcal{K}, \mathcal{H})$. We now recall the main concepts from [17] for the convenience of the reader.

Definition 2.2. Let $B \in \mathcal{L}(\mathcal{K}, \mathcal{H})$ and $\widetilde{B} \in \mathcal{L}(\mathcal{H}, \mathcal{K})$. We define extensions of $A$ and $\widetilde{A}$ (respectively) by

$$
A_{B}:=\left.\widetilde{A}^{*}\right|_{\operatorname{ker}\left(\Gamma_{1}-B \Gamma_{2}\right)} \quad \text { and } \quad \widetilde{A}_{\widetilde{B}}:=\left.A^{*}\right|_{\operatorname{ker}\left(\widetilde{\Gamma}_{1}-\widetilde{B} \widetilde{\Gamma}_{2}\right)} .
$$

In the following, we assume $\rho\left(A_{B}\right) \neq \emptyset$, in particular $A_{B}$ is a closed operator. For $\lambda \in \rho\left(A_{B}\right)$, we define the $M$-function via $M_{B}(\lambda): \operatorname{Ran}\left(\Gamma_{1}-B \Gamma_{2}\right) \rightarrow \mathcal{K}$ :

$$
M_{B}(\lambda)\left(\Gamma_{1}-B \Gamma_{2}\right) u=\Gamma_{2} u \quad \text { for all } u \in \operatorname{ker}\left(\widetilde{A}^{*}-\lambda\right),
$$

and for $\lambda \in \rho\left(\widetilde{A}_{\widetilde{B}}\right)$, we define $\widetilde{M}_{\widetilde{B}}(\lambda): \operatorname{Ran}\left(\widetilde{\Gamma}_{1}-\widetilde{B}_{\Gamma_{2}}\right) \rightarrow \mathcal{H}$ by

$$
\widetilde{M}_{\widetilde{B}}(\lambda)\left(\widetilde{\Gamma}_{1}-\widetilde{B} \widetilde{\Gamma}_{2}\right) v=\widetilde{\Gamma}_{2} v \quad \text { for all } v \in \operatorname{ker}\left(A^{*}-\lambda\right) .
$$

It will follow from Lemma 2.5 that $M_{B}(\lambda)$ and $\widetilde{M}_{\widetilde{B}}(\lambda)$ are well defined for $\lambda \in \rho\left(A_{B}\right)$ and $\lambda \in \rho\left(\widetilde{A}_{\widetilde{B}}\right)$, respectively. Moreover, in our situation $\operatorname{Ran}\left(\Gamma_{1}-B \Gamma_{2}\right)=\mathcal{H}$ and $\operatorname{Ran}\left(\widetilde{\Gamma}_{1}-\widetilde{B} \widetilde{\Gamma}_{2}\right)=\mathcal{K}$, so the $M$-functions are defined on the whole spaces.

Definition 2.3 (Solution operator). For $\lambda \in \rho\left(A_{B}\right)$, we define the linear operator $S_{\lambda, B}: \operatorname{Ran}\left(\Gamma_{1}-B \Gamma_{2}\right) \rightarrow \operatorname{ker}\left(\widetilde{A}^{*}-\lambda\right)$ by

$$
\left(\widetilde{A}^{*}-\lambda\right) S_{\lambda, B} f=0, \quad\left(\Gamma_{1}-B \Gamma_{2}\right) S_{\lambda, B} f=f,
$$


i.e. $S_{\lambda, B}=\left(\left.\left(\Gamma_{1}-B \Gamma_{2}\right)\right|_{\operatorname{ker}\left(\widetilde{A}^{*}-\lambda\right)}\right)^{-1}$. For $\lambda \in \rho\left(\widetilde{A}_{B}^{*}\right)$, we define the linear operator $\widetilde{S}_{\lambda, B^{*}}: \operatorname{Ran}\left(\widetilde{\Gamma}_{1}-B^{*} \widetilde{\Gamma}_{2}\right) \rightarrow \operatorname{ker}\left(A^{*}-\lambda\right)$ by

$$
\left(A^{*}-\lambda\right) \widetilde{S}_{\lambda, B^{*}} f=0, \quad\left(\widetilde{\Gamma}_{1}-B^{*} \widetilde{\Gamma}_{2}\right) \widetilde{S}_{\lambda, B^{*}} f=f .
$$

All the following results have a corresponding version for the quantities $\widetilde{M}_{\widetilde{B}}, \widetilde{S}_{\lambda, B^{*}}$ etc. obtained from the formally adjoint problem.

REMARK 2.4. (1) As we are not interested in characterizing all closed extensions of $A$, in this paper we will assume for simplicity that $B \in \mathcal{L}(\mathcal{K}, \mathcal{H})$. A discussion of all closed extensions of $A$ in the boundary triple setting can be found in [15].

(2) Note that $M_{B}(\lambda)=\Gamma_{2} S_{\lambda, B}$.

(3) $M$-functions with different boundary conditions are related by the Aronszajn-Donoghue formula (cf. also (2.12)):

$$
M_{B}(\lambda)=\left(I+M_{B}(\lambda)(B-C)\right) M_{C}(\lambda)=M_{C}(\lambda)\left(I+(B-C) M_{B}(\lambda)\right) .
$$

The following lemma summarizes [16, Lemma 2.4 and Corollary 2.5].

LEMMA 2.5 .

(1) $S_{\lambda, B}$ is well-defined for $\lambda \in \rho\left(A_{B}\right)$.

(2) For each $f \in \operatorname{Ran}\left(\Gamma_{1}-B \Gamma_{2}\right)$ the map from $\rho\left(A_{B}\right)$ to $H$ given by $\lambda \mapsto S_{\lambda, B} f$ is analytic.

(3) For $\lambda, \lambda_{0} \in \rho\left(A_{B}\right)$ we have $S_{\lambda, B}=S_{\lambda_{0}, B}+\left(\lambda-\lambda_{0}\right)\left(A_{B}-\lambda\right)^{-1} S_{\lambda_{0}, B}$.

The difference of two resolvents can be related to the $M$-function by Krein-type resolvent formulae, such as

$$
\begin{aligned}
\left(A_{C}-\lambda\right)^{-1}- & \left(A_{B}-\lambda\right)^{-1} \\
& =S_{\lambda, C}\left(I+(B-C) M_{B}(\lambda)\right)\left(\Gamma_{1}-B \Gamma_{2}\right)\left(A_{C}-\lambda\right)^{-1} \\
& =S_{\lambda, C}\left(I+(B-C) M_{B}(\lambda)\right)(C-B) \Gamma_{2}\left(A_{C}-\lambda\right)^{-1}
\end{aligned}
$$

for $B, C \in \mathcal{L}(\mathcal{K}, \mathcal{H})$ and $\lambda \in \rho\left(A_{B}\right) \cap \rho\left(A_{C}\right)$ (see [16, Theorem 2.6]).

The following formula already appears in some proofs in $[16,17]$, but due to its importance in our later analysis, we state it here.

Lemma 2.6. For all $F \in D\left(\widetilde{A}^{*}\right), v \in D\left(A^{*}\right)$ and $\lambda \in \rho\left(A_{B}\right)$ we have

$$
\begin{aligned}
\left\langle F-\left(A_{B}-\lambda\right)^{-1}\left(\widetilde{A}^{*}-\lambda\right) F,\left(A^{*}-\bar{\lambda} I\right) v\right\rangle & \\
= & \left\langle M_{B}(\lambda) f,\left(\widetilde{\Gamma}_{1}-B^{*} \widetilde{\Gamma}_{2}\right) v\right\rangle_{\mathcal{K}}-\left\langle f, \widetilde{\Gamma}_{2} v\right\rangle_{\mathcal{H}}
\end{aligned}
$$

where $f=\left(\Gamma_{1}-B \Gamma_{2}\right) F$.

We are now ready to define one of the main concepts of the paper, the detectable subspaces, introduced in [16]. 
Definition 2.7. Fix $\mu_{0} \notin \sigma\left(A_{B}\right)$. We define the spaces

$$
\begin{aligned}
\mathcal{S}_{B} & =\operatorname{Span}_{\delta \notin \sigma\left(A_{B}\right)}\left(A_{B}-\delta I\right)^{-1} \operatorname{Ran} S_{\mu_{0}, B}, \\
\mathcal{T}_{B} & =\operatorname{Span}_{\mu \notin \sigma\left(A_{B}\right)} \operatorname{Ran} S_{\mu, B},
\end{aligned}
$$

and similarly

$$
\begin{aligned}
\widetilde{\mathcal{S}}_{B^{*}} & =\operatorname{Span}_{\delta \notin \sigma\left(\widetilde{A}_{B^{*}}\right)}\left(\widetilde{A}_{B^{*}}-\delta I\right)^{-1} \operatorname{Ran} \widetilde{S}_{\widetilde{\mu}, B^{*}}, \\
\widetilde{\mathcal{T}}_{B^{*}} & =\operatorname{Span}_{\mu \notin \sigma\left(\widetilde{A}_{B^{*}}\right)} \operatorname{Ran} \widetilde{S}_{\mu, B^{*}} .
\end{aligned}
$$

We call $\overline{\mathcal{S}_{B}}$ and $\overline{\widetilde{\mathcal{S}}_{B^{*}}}$ the detectable subspaces.

REMARK 2.8. Note that the definition of the detectable subspace is reminiscent of the notion of complete non-selfadjointness of an operator in Hilbert space [49]. The Langer decomposition of an operator into its selfadjoint and completely non-selfadjoint reducing parts is similar to the reduction of our operator to the detectable subspace. Indeed, in (2.8) we develop by the resolvent of $A_{B}$ the range of a solution operator, while in the construction of the "completely non-selfadjoint subspace" one has to develop the range of the "imaginary part" of an operator by its resolvent.

We now consider the dependence of these spaces on $\mu_{0}$ and $B$.

Proposition 2.9.

(1) Let $B \in \mathcal{L}(\mathcal{K}, \mathcal{H})$. Assume that there is a sequence $\left(z_{n}\right)_{n \in \mathbb{N}}$ in $\mathbb{C}$ with $\left|z_{n}\right| \rightarrow \infty$ and $\left(\left\|z_{n}\left(A_{B}-z_{n} I\right)^{-1}\right\|\right)_{n \in \mathbb{N}}$ bounded. Then $\overline{\mathcal{S}_{B}}=\overline{\mathcal{T}_{B}}$. In particular, $\overline{\mathcal{S}_{B}}$ is independent of $\mu_{0}$.

(2) Let $B, C \in \mathcal{L}(\mathcal{K}, \mathcal{H})$. If $\rho\left(A_{B}\right) \cup \rho\left(A_{C}\right) \subseteq \overline{\rho\left(A_{B}\right) \cap \rho\left(A_{C}\right)}$, then $\overline{\mathcal{T}_{B}}=\overline{\mathcal{T}_{C}}$.

(3) Suppose that for all $B, C \in \mathcal{L}(\mathcal{K}, \mathcal{H})$, we have

$$
\rho\left(A_{B}\right) \cup \rho\left(A_{C}\right) \subseteq \overline{\rho\left(A_{B}\right) \cap \rho\left(A_{C}\right)} .
$$

Then $\overline{\mathcal{T}_{B}}=\overline{\operatorname{Span}_{\lambda \in \Lambda} \operatorname{ker}\left(\widetilde{A}^{*}-\lambda\right)}$, where $\Lambda=\bigcup_{C \in \mathcal{L}(\mathcal{K}, \mathcal{H})} \rho\left(A_{C}\right)$.

Proof. (1) This is shown in [16, Lemma 3.1].

(2) From [17, Proposition 4.5] we have

$$
S_{\lambda, C}\left(I-(C-B) \Gamma_{2} S_{\lambda, B}\right)=S_{\lambda, B},
$$

we note that $\operatorname{Ran} S_{\lambda, B}=\operatorname{Ran} S_{\lambda, C}$ whenever $\lambda \in \rho\left(A_{B}\right) \cap \rho\left(A_{C}\right)$. Now assume $\lambda \in \rho\left(A_{B}\right) \cap \sigma\left(A_{C}\right)$. We need to show that $\operatorname{Ran} S_{\lambda, B} \subseteq \overline{\mathcal{T}^{\prime}}$ where

$$
\mathcal{T}^{\prime}=\underset{\mu \in \rho\left(A_{B}\right) \cap \rho\left(A_{C}\right)}{\operatorname{Span}} \operatorname{Ran} S_{\mu, B}
$$


By assumption, there exists a sequence $\left(\lambda_{n}\right)_{n \in \mathbb{N}}$ in $\rho\left(A_{B}\right) \cap \rho\left(A_{C}\right)$ with $\lambda_{n} \rightarrow \lambda$. Let $u=S_{\lambda, B} f$. We have

$$
S_{\lambda_{n}, B}-S_{\lambda, B}=\left(\lambda_{n}-\lambda\right)\left(A_{B}-\lambda_{n}\right)^{-1} S_{\lambda, B} .
$$

Therefore,

$$
\left\|S_{\lambda_{n}, B} f-S_{\lambda, B} f\right\| \leq\left|\lambda_{n}-\lambda\right|\left\|\left(A_{B}-\lambda_{n}\right)^{-1}\right\|\left\|S_{\lambda, B} f\right\| .
$$

As $n \rightarrow \infty,\left\|\left(A_{B}-\lambda_{n}\right)^{-1}\right\| \rightarrow\left\|\left(A_{B}-\lambda\right)^{-1}\right\|<\infty$, so $S_{\lambda_{n}, B} f \rightarrow S_{\lambda, B} f$, which completes the proof.

(3) This follows immediately from the previous part of the proposition.

REMARK 2.10. We note that the conditions in parts (1) and (3) of the proposition are satisfied in many interesting cases, in particular that of 'weak' perturbations of selfadjoint operators.

Throughout the remainder of this article, we will assume that the spaces $\overline{\mathcal{S}_{B}}$ and $\overline{\mathcal{T}_{B}}$ coincide, are independent of $B$ and equal $\overline{\operatorname{Span}_{\lambda \in \Lambda} \operatorname{ker}\left(\widetilde{A}^{*}-\lambda\right)}$. To avoid cumbersome notation, we shall denote all these spaces by $\overline{\mathcal{S}}$. We shall also denote $\mathcal{S}_{B}$ by $\mathcal{S}$ and $\mathcal{T}_{B}$ by $\mathcal{T}$ when no confusion can arise. We will refer to $\overline{\mathcal{S}}$ as the detectable subspace.

In [16, Lemma 3.4], it is shown that $\overline{\mathcal{S}}$ is a regular invariant space of the resolvent of the operator $A_{B}$, that is, $\overline{\left(A_{B}-\mu I\right)^{-1} \overline{\mathcal{S}}}=\overline{\mathcal{S}}$ for all $\mu \in \rho\left(A_{B}\right)$.

From (2.8) and Proposition 2.9(3), we get

$$
\mathcal{S}^{\perp}=\bigcap_{B, \lambda \in \rho\left(A_{B}\right)} \operatorname{ker} S_{\lambda, B}^{*}
$$

Moreover, from [17, Proposition 3.9] we have

$$
\operatorname{ker} S_{\lambda, B}^{*}=\operatorname{ker}\left(\widetilde{\Gamma}_{2}\left(\widetilde{A}_{B^{*}}-\bar{\lambda}\right)^{-1}\right) .
$$

Now assume that $h \in \mathcal{S}^{\perp}$. Then $\widetilde{\Gamma}_{2}\left(\widetilde{A}_{B^{*}}-\bar{\lambda}\right)^{-1} h=0$ for all suitable $B$ and $\lambda$. Fixing $B$ and $\lambda$ and setting

$$
y_{B}=\left(\widetilde{A}_{B^{*}}-\bar{\lambda}\right)^{-1} h,
$$

we get $\widetilde{\Gamma}_{2} y_{B}=0$ and hence $\widetilde{\Gamma}_{1} y_{B}=B^{*} \widetilde{\Gamma}_{2} y_{B}=0$, so $y_{B}$ satisfies any homogeneous boundary condition and lies in the domain of the minimal operator. Hence,

$$
\mathcal{S}^{\perp}=\left\{h \in H: \forall B^{*}, \lambda \in \rho\left(\widetilde{A}_{B^{*}}\right), \widetilde{\Gamma}_{i}\left(\widetilde{A}_{B^{*}}-\lambda\right)^{-1} h=0 \text { for } i=1,2\right\} .
$$

REMARK 2.11. Determining the detectable subspace $\mathcal{S}$ is closely related to the problem of observability in systems theory. Indeed, from (2.13) and (2.15) the space $\mathcal{S}^{\perp}$ coincides (at least formally) with the 'non-observable for all time' subspace $N(\Theta)$ of a system $\Theta=(A, B, C, D)$ (see [46]), in which $A=\widetilde{A}_{B^{*}}, B=\widetilde{\mathcal{S}}_{\lambda_{0}, B^{*}}$ for some $\lambda_{0} \in \rho\left(\widetilde{A}_{B^{*}}\right), C=\widetilde{\Gamma}_{2}\left(\widetilde{A}^{*}-\lambda_{0}\right), D=0$, though there are several differences between these notions: 
(1) The corresponding system can be highly awkward to construct and involves unbounded operators.

(2) In systems theory the subspace of non-observable states is generated by the resolvent in one half-plane only (corresponding to positive times $t$ only). In our construction, the spectral parameter runs through the whole resolvent set. It is known that when the resolvent set consists of several disconnected domains, developing the linear set by the resolvent essentially depends on the choice of component.

(3) We do not require the operator $\widetilde{A}_{B^{*}}$ to be the generator of a semigroup. In particular, the resolvent set may have a complicated geometrical structure. If $\widetilde{A}_{B^{*}}$ is a generator, the resolvent in (2.15) can be replaced by the positive and negative time semigroups.

Despite these differences, the similarity to the observability problem may be fruitful for analysing detectability. For more connections between boundary triples and systems theory, we refer to [45].

3. Three concrete applications. In this section we examine three different operators which will be used to illustrate the power and also the limitations of the theory. For the first example we show that $\overline{\mathcal{S}}$ is the whole underlying Hilbert space; for the second we refer the reader to some previous work, which shows that $\overline{\mathcal{S}}$ may or may not be the whole space; for the third example, we present the $M$-function and the resolvent, in preparation for the more substantial work in Section 7, which shows that the characterization of $\overline{\mathcal{S}}$ may be very subtle for this seemingly innocuous model.

3.1. Schrödinger problems. For complex-valued $q \in L^{\infty}(0,1)$, consider

$$
L u=\left(-\frac{d^{2}}{d x^{2}}+q\right) u \quad \text { and } \quad \widetilde{L} u=\left(-\frac{d^{2}}{d x^{2}}+\bar{q}\right) u \quad \text { on }[0,1] .
$$

Let $A u=L u$ and $\widetilde{A} u=\widetilde{L} u$ with $D(A)=D(\widetilde{A})=H_{0}^{2}(0,1)$. Then $\widetilde{A}^{*} u=L u$ and $A^{*} u=\widetilde{L} u$ with $D\left(\widetilde{A}^{*}\right)=D\left(A^{*}\right)=H^{2}(0,1)$, and for $u, v \in H^{2}(0,1)$,

$$
\left\langle\widetilde{A}^{*} u, v\right\rangle-\left\langle u, A^{*} v\right\rangle=\left\langle\Gamma_{1} u, \Gamma_{2} v\right\rangle-\left\langle\Gamma_{2} u, \Gamma_{1} v\right\rangle
$$

where

$$
\Gamma_{1} u=\left(\begin{array}{c}
-u^{\prime}(1) \\
u^{\prime}(0)
\end{array}\right), \quad \Gamma_{2} u=\left(\begin{array}{c}
u(1) \\
u(0)
\end{array}\right) .
$$

In particular, $\Gamma_{1}=\widetilde{\Gamma}_{1}, \Gamma_{2}=\widetilde{\Gamma}_{2}$ and $\mathcal{H}=\mathcal{K}=\mathbb{C}^{2}$.

Then the following result is proved in [18, Section 3.1]:

Proposition 3.1. For the Schrödinger operator we have $\overline{\mathcal{S}}=L^{2}(0,1)$. 
Similarly, the result that the detectable subspace is the whole space is true for Schrödinger operators on the whole line or half-line, even with operator-valued coefficients [26] and for higher-dimensional cases [12].

\subsection{Hain-Lüst-type operators. Let}

$$
\widetilde{A}^{*}=\left(\begin{array}{cc}
-\frac{d^{2}}{d x^{2}}+q(x) & \tilde{w}(x) \\
w(x) & u(x)
\end{array}\right) \quad \text { and } \quad A^{*}=\left(\begin{array}{cc}
-\frac{d^{2}}{d x^{2}}+\overline{q(x)} & \overline{w(x)} \\
\overline{\tilde{w}(x)} & \overline{u(x)}
\end{array}\right),
$$

where $q, u, \tilde{w}$ and $w$ are $L^{\infty}$-functions, and the domain of the operators is given by

$$
D\left(\widetilde{A}^{*}\right)=D\left(A^{*}\right)=H^{2}(0,1) \times L^{2}(0,1) .
$$

It is then easy to see that

$$
\begin{aligned}
\left\langle\widetilde{A}^{*}\left(\begin{array}{l}
y \\
z
\end{array}\right),\left(\begin{array}{l}
f \\
g
\end{array}\right)\right\rangle-\left\langle\left(\begin{array}{l}
y \\
z
\end{array}\right)\right. & \left.A^{*}\left(\begin{array}{l}
f \\
g
\end{array}\right)\right\rangle \\
& =\left\langle\Gamma_{1}\left(\begin{array}{l}
y \\
z
\end{array}\right), \Gamma_{2}\left(\begin{array}{l}
f \\
g
\end{array}\right)\right\rangle-\left\langle\Gamma_{2}\left(\begin{array}{l}
y \\
z
\end{array}\right), \Gamma_{1}\left(\begin{array}{l}
f \\
g
\end{array}\right)\right\rangle,
\end{aligned}
$$

where

$$
\mathcal{H}=\mathcal{K}=\mathbb{C}^{2}, \quad \Gamma_{1}\left(\begin{array}{l}
y \\
z
\end{array}\right)=\left(\begin{array}{c}
-y^{\prime}(1) \\
y^{\prime}(0)
\end{array}\right), \quad \Gamma_{2}\left(\begin{array}{l}
y \\
z
\end{array}\right)=\left(\begin{array}{l}
y(1) \\
y(0)
\end{array}\right) .
$$

Some information on $\mathcal{S}$ for these operators is available in [16]. In particular we show there that if $w=\tilde{w}$ then $\overline{\mathcal{S}} \subseteq L^{2}(0,1) \oplus L^{2}\left(w^{-1}(\{0\})^{c}\right)$ (where $\Omega^{c}$ denotes the complement of a set $\Omega$ ) and so if $w$ vanishes on a set of positive measure then $\overline{\mathcal{S}}$ is not the whole underlying space. A more detailed investigation of detectable subspaces for Hain-Lüst-type operators can be found in [18]. In particular, a full analysis of the detectable subspace for the case $w \tilde{w}=0$ is developed there.

3.3. The Friedrichs model. We consider in $L^{2}(\mathbb{R})$ the operator $A$ with domain $D(A)$ equal to the set

(3.4) $\left\{f \in L^{2}(\mathbb{R}): x f(x) \in L^{2}(\mathbb{R}), \lim _{R \rightarrow \infty} \int_{-R}^{R} f(x) d x\right.$ exists and is zero $\}$, and given by the expression

$$
(A f)(x)=x f(x)+\langle f, \phi\rangle \psi(x),
$$

where $\phi, \psi$ are in $L^{2}(\mathbb{R})$. Observe that since the constant function 1 does not lie in $L^{2}(\mathbb{R})$, the domain of $A$ is dense.

We first collect some results from [16], where more details and proofs can be found: 
Lemma 3.2. The adjoint of $A$ is given on the domain

$$
D\left(A^{*}\right)=\left\{f \in L^{2}(\mathbb{R}): \exists c_{f} \in \mathbb{C}: x f(x)-c_{f} \mathbf{1} \in L^{2}(\mathbb{R})\right\}
$$

by the formula

$$
A^{*} f=x f(x)-c_{f} \mathbf{1}+\langle f, \psi\rangle \phi .
$$

Since $c_{f}=\lim _{R \rightarrow \infty}(2 R)^{-1} \int_{-R}^{R} x f(x) d x$ is uniquely determined, we can define trace operators $\Gamma_{1}$ and $\Gamma_{2}$ on $D\left(A^{*}\right)$ as follows:

$$
\Gamma_{1} u=\lim _{R \rightarrow \infty} \int_{-R}^{R} u(x) d x, \quad \Gamma_{2} u=c_{u} .
$$

Note that $\Gamma_{1} u=\int_{\mathbb{R}}\left(u(x)-c_{u} \mathbf{1} \operatorname{sign}(x)\left(x^{2}+1\right)^{-1 / 2}\right) d x$, which is the expression used in [16].

Lemma 3.3. The operators $\Gamma_{1}$ and $\Gamma_{2}$ are bounded relative to $A^{*}$ and the following 'Green identity' holds:

$$
\left\langle A^{*} f, g\right\rangle-\left\langle f, A^{*} g\right\rangle=\Gamma_{1} f \overline{\Gamma_{2} g}-\Gamma_{2} f \overline{\Gamma_{1} g}+\langle f, \psi\rangle\langle\phi, g\rangle-\langle f, \phi\rangle\langle\psi, g\rangle .
$$

We introduce an operator $\widetilde{A}$ in which the roles of $\phi$ and $\psi$ are exchanged: $D(\widetilde{A})=D(A)$ and

$$
(\widetilde{A} f)(x)=x f(x)+\langle f, \psi\rangle \phi(x) .
$$

In view of Lemma 3.2 we immediately see that $D\left(\widetilde{A}^{*}\right)=D\left(A^{*}\right)$ and that

$$
\left(\widetilde{A}^{*} f\right)(x)=x f(x)-c_{f} \mathbf{1}+\langle f, \phi\rangle \psi(x) .
$$

Thus $\widetilde{A}^{*}$ is an extension of $A, A^{*}$ is an extension of $\widetilde{A}$, and the following result is easily proved.

LEMMA 3.4. We have

$$
A=\left.\widetilde{A}^{*}\right|_{\operatorname{ker} \Gamma_{1} \cap \operatorname{ker} \Gamma_{2}} \quad \text { and } \quad \widetilde{A}=\left.A^{*}\right|_{\operatorname{ker} \Gamma_{1} \cap \operatorname{ker} \Gamma_{2}} ;
$$

moreover, the Green formula (3.9) can be modified to

$$
\left\langle A^{*} f, g\right\rangle-\left\langle f, \widetilde{A}^{*} g\right\rangle=\Gamma_{1} f \overline{\Gamma_{2} g}-\Gamma_{2} f \overline{\Gamma_{1} g} .
$$

We finish our review from [16] with the $M$-function and the resolvent:

Lemma 3.5. Suppose that $\Im \lambda \neq 0$. Then $f \in \operatorname{ker}\left(\widetilde{A}^{*}-\lambda\right)$ if

$$
f(x)=\Gamma_{2} f\left[\frac{1}{x-\lambda}-\frac{\left\langle(t-\lambda)^{-1}, \phi\right\rangle}{D(\lambda)} \frac{\psi(x)}{x-\lambda}\right] .
$$

Here

$$
D(\lambda)=1+\int_{\mathbb{R}} \frac{1}{x-\lambda} \psi(x) \overline{\phi(x)} d x
$$


Moreover the Titchmarsh-Weyl coefficient $M_{B}(\lambda)$ is given by

$$
M_{B}(\lambda)=\left[\operatorname{sign}(\Im \lambda) \pi i-\frac{\left\langle(t-\lambda)^{-1}, \bar{\psi}\right\rangle\left\langle(t-\lambda)^{-1}, \phi\right\rangle}{D(\lambda)}-B\right]^{-1} .
$$

For the resolvent, we have $\left(A_{B}-\lambda\right) f=g$ if and only if

$$
\begin{aligned}
f(x)= & \frac{g(x)}{x-\lambda}-\frac{1}{D(\lambda)} \frac{\psi(x)}{x-\lambda}\left\langle\frac{g}{t-\lambda}, \phi\right\rangle \\
& +c_{f}\left[\frac{1}{x-\lambda}-\frac{1}{D(\lambda)} \frac{\psi(x)}{x-\lambda}\left\langle\frac{1}{t-\lambda}, \phi\right\rangle\right]
\end{aligned}
$$

with

$$
c_{f}=M_{B}(\lambda)\left[-\left\langle\frac{1}{t-\lambda}, \bar{g}\right\rangle+\frac{1}{D(\lambda)}\left\langle\frac{g}{t-\lambda}, \phi\right\rangle\left\langle\frac{1}{t-\lambda}, \bar{\psi}\right\rangle\right] .
$$

These expressions will be needed in Section 7 .

REMARK 3.6. There is another approach to the Friedrichs model, via Fourier transforms, which may appear more natural. This turns the multiplication operator into a first order differential operator, while the boundary operators are given in terms of the right and left limits at 0 (see [19]).

4. Relation between $M$-function and resolvent on $\overline{\mathcal{S}}$. Having introduced some concrete examples in the previous section, we now turn our attention to what can be shown in the general setting. Our aim is to study the relation between $M_{B}(\lambda)$ and the bordered resolvent $\left.P_{\widetilde{\mathcal{S}}}\left(A_{B}-\lambda\right)^{-1}\right|_{\overline{\mathcal{S}}}$ where for any subspace $M, P_{M}$ denotes the orthogonal projection onto $M$.

\subsection{Information on the $M$-function contained in a single resol-} vent. We first look at gaining information on the $M$-function from knowledge of the resolvent for a single boundary-condition operator $B$. Our results are of two types, concerning uniqueness and reconstruction. The distinction lies in the fact that the proof of a uniqueness result may not provide a constructive procedure for recovering the $M$-function. Reconstruction results are stronger: the Gel'fand-Levitan approach to inverse Schrödinger problems, for instance, consists of a reconstruction result, using integral equations. In the abstract setting considered here, we encounter cases for which we have a uniqueness result but no reconstruction procedure.

Our first theorem concerns uniqueness only.

Theorem 4.1. Let $\lambda \in \rho\left(A_{B}\right)$. Then $\left.P_{\overline{\widetilde{\mathcal{S}}}}\left(A_{B}-\lambda\right)^{-1}\right|_{\overline{\mathcal{S}}}$ uniquely determines $M_{B}(\lambda)$. In particular, if also $\lambda \in \rho\left(A_{C}\right)$, then $\left.P_{\widetilde{\mathcal{S}}}\left(A_{B}-\lambda\right)^{-1}\right|_{\overline{\mathcal{S}}}=$ $\left.P_{\overline{\widetilde{\mathcal{S}}}}\left(A_{C}-\lambda\right)^{-1}\right|_{\overline{\mathcal{S}}}$ implies that $M_{B}(\lambda)=M_{C}(\lambda)$, and if additionally $\lambda \in$ $\rho\left(A_{\infty}\right)$, then $B=C$. Here, $A_{\infty}=\left.\widetilde{A}^{*}\right|_{\operatorname{ker} \Gamma_{2}}$. 
Proof. Assume $\widehat{M}_{B}(\lambda)$ is a different $M$-function for the same problem. By surjectivity of the trace operators there exist $F \in D\left(\widetilde{A}^{*}\right)$ and $v \in D\left(A^{*}\right)$ such that

$\left\langle M_{B}(\lambda)\left(\Gamma_{1}-B \Gamma_{2}\right) F,\left(\widetilde{\Gamma}_{1}-B^{*} \widetilde{\Gamma}_{2}\right) v\right\rangle_{\mathcal{K}} \neq\left\langle\widehat{M}_{B}(\lambda)\left(\Gamma_{1}-B \Gamma_{2}\right) F,\left(\widetilde{\Gamma}_{1}-B^{*} \widetilde{\Gamma}_{2}\right) v\right\rangle_{\mathcal{K}}$.

Setting $h=S_{\mu, B}\left(\Gamma_{1}-B \Gamma_{2}\right) F \in \mathcal{S}$ and $\widetilde{h}=\widetilde{S}_{\widetilde{\mu}, B^{*}}\left(\widetilde{\Gamma}_{1}-B^{*} \widetilde{\Gamma}_{2}\right) v \in \widetilde{\mathcal{S}}$ and using (2.7), we find that

$$
\left\langle\left(I-\left(A_{B}-\lambda\right)^{-1}(\mu-\lambda)\right) h,(\widetilde{\mu}-\bar{\lambda}) \widetilde{h}\right\rangle
$$

has two different values. Therefore, $\left\langle\left(A_{B}-\lambda\right)^{-1} h, \widetilde{h}\right\rangle$ has two different values, yielding a contradiction.

If $\left.P_{\widetilde{\mathcal{S}}}\left(A_{B}-\lambda\right)^{-1}\right|_{\overline{\mathcal{S}}}=\left.P_{\overline{\widetilde{\mathcal{S}}}}\left(A_{C}-\lambda\right)^{-1}\right|_{\overline{\mathcal{S}}}$, then by the argument above $\left\langle M_{B}(\lambda)\left(\Gamma_{1}-B \Gamma_{2}\right) F,\left(\widetilde{\Gamma}_{1}-B^{*} \widetilde{\Gamma}_{2}\right) v\right\rangle_{\mathcal{K}}=\left\langle M_{C}(\lambda)\left(\Gamma_{1}-B \Gamma_{2}\right) F,\left(\widetilde{\Gamma}_{1}-B^{*} \widetilde{\Gamma}_{2}\right) v\right\rangle_{\mathcal{K}}$. Choosing $F$ and $v$ such that $\Gamma_{2} F=\widetilde{\Gamma}_{2} v=0$ and $\Gamma_{1} F$ and $\widetilde{\Gamma}_{1} v$ are arbitrary, we obtain

$$
\left\langle M_{B}(\lambda) \Gamma_{1} F, \widetilde{\Gamma}_{1} v\right\rangle_{\mathcal{K}}=\left\langle M_{C}(\lambda) \Gamma_{1} F, \widetilde{\Gamma}_{1} v\right\rangle_{\mathcal{K}}
$$

so $M_{B}(\lambda)=M_{C}(\lambda)$. If $\lambda \in \rho\left(A_{\infty}\right)$, then $\operatorname{Ran} M_{B}(\lambda)=\mathcal{K}$ and $\operatorname{ker} M_{C}(\lambda)$ $=\{0\}$, so from $(2.5)$ we get $B=C$.

In the case of the Friedrichs model, we shall show in a forthcoming paper that reconstruction of $M_{B}(\lambda)$ is also possible under the hypotheses of Theorem 4.1. In the completely abstract case, so far, we have only been able to develop a reconstruction method to recover $M_{B}(\lambda)$ from one bordered resolvent on $\overline{\mathcal{S}}$ if some additional information is added. Note that from the knowledge of the resolvent and the range of one solution operator we can explicitly reconstruct $\overline{\mathcal{S}}$. More precisely, we have the following result.

TheOrem 4.2. Assume we know $\overline{\mathcal{S}},\left.P_{\overline{\widetilde{\mathcal{S}}}}\left(A_{B}-\lambda\right)^{-1}\right|_{\overline{\mathcal{S}}}$ and the two sets $\overline{\operatorname{Ran} S_{\mu, B}}, \overline{\operatorname{Ran} \widetilde{S}_{\widetilde{\mu}, B^{*}}}$ for some $(\mu, \widetilde{\mu})$ with $\mu, \overline{\widetilde{\mu}} \in \rho\left(A_{B}\right)$. Then we can reconstruct $M_{B}(\lambda)$ uniquely if $B$ is known.

Proof. For $h \in \overline{\operatorname{Ran} S_{\mu, B}}$ and $\widetilde{h} \in \overline{\operatorname{Ran} \widetilde{S}_{\widetilde{\mu}, B^{*}}}$, consider

$$
H(h, \widetilde{h})=\left\langle\left(I-\left(A_{B}-\lambda\right)^{-1}(\mu-\lambda)\right) h,(\widetilde{\mu}-\bar{\lambda}) \widetilde{h}\right\rangle .
$$

By assumption, we know $H(h, \widetilde{h})$. Varying $h$ in $\overline{\operatorname{Ran} S_{\mu, B}}$, we see that $\left(\Gamma_{1}-B \Gamma_{2}\right) h$ runs through the whole of $\mathcal{H}$; if we vary $\widetilde{h}$ in $\overline{\operatorname{Ran} \widetilde{S}_{\widetilde{\mu}, B^{*}}}$, the values $\left(\widetilde{\Gamma}_{1}-B^{*} \widetilde{\Gamma}_{2}\right) \widetilde{h}$ run through the whole of $\mathcal{K}$ and $\widetilde{\Gamma}_{2} \widetilde{h}$ through the whole of $\mathcal{H}$. Using Lemma 2.6 we deduce for a dense set of $h, \tilde{h}$ that

$$
H(h, \widetilde{h})=\left\langle M_{B}(\lambda)\left(\Gamma_{1}-B \Gamma_{2}\right) h,\left(\widetilde{\Gamma}_{1}-B^{*} \widetilde{\Gamma}_{2}\right) \widetilde{h}\right\rangle_{\mathcal{K}}-\left\langle\left(\Gamma_{1}-B \Gamma_{2}\right) h, \widetilde{\Gamma}_{2} \widetilde{h}\right\rangle_{\mathcal{H}},
$$

which allows reconstruction of the $M$-function. 
A key hypothesis of Theorem 4.2 is the assumption that we know the closed ranges of the solution operators. We now examine how strong a hypothesis this may be, considering first the Friedrichs model.

Proposition 4.3. Assume we know $\operatorname{Ran} S_{\lambda, B}$ and $\operatorname{Ran} \widetilde{S}_{\mu, B^{*}}$ for some $\lambda, \mu$ for the Friedrichs model. Moreover, assume $\operatorname{Ran} S_{\lambda, B} \neq \operatorname{Span}\left\{\frac{1}{x-\lambda}\right\}$ and $\operatorname{Ran} \widetilde{S}_{\mu, B^{*}} \neq \operatorname{Span}\left\{\frac{1}{x-\mu}\right\}$ (which is true for generic $\phi, \psi$ ). Then the operator is uniquely determined.

Proof. Assume we know $\operatorname{Ran} S_{\lambda, B}$ and $\operatorname{Ran} \widetilde{S}_{\mu, B^{*}}$ for some $\lambda, \mu$. Choose $u$ and $v$ in the ranges with $c_{u}=1$ and $c_{v}=1$; from (3.14) we have

$$
\begin{aligned}
& u(x)=\frac{1}{x-\lambda}-\frac{\left\langle(t-\lambda)^{-1}, \phi\right\rangle}{D(\lambda)} \frac{\psi(x)}{x-\lambda} \\
& v(x)=\frac{1}{x-\mu}-\frac{\left\langle(t-\mu)^{-1}, \psi\right\rangle}{\overline{D(\mu)}} \frac{\phi(x)}{x-\mu} .
\end{aligned}
$$

As one of the functions $\phi, \psi$ is only determined up to a scalar factor, we may normalize $\phi$ so that $\left\langle(t-\lambda)^{-1}, \phi\right\rangle=D(\lambda)$. This allows us to determine $\psi$ from the first expression, which also gives us $\left\langle(t-\mu)^{-1}, \psi\right\rangle$, so $\phi(x) / \overline{D(\mu)}$ is known. Solving $v(x)$ for $\phi(x)$, we get

$$
\phi(x)=(1-(x-\mu) v(x)) \frac{\overline{D(\mu)}}{\left\langle(t-\mu)^{-1}, \psi\right\rangle} .
$$

Multiplying by $\overline{(x-\lambda)^{-1} D(\lambda)^{-1}}$ and integrating in $x$, we can determine the product $D(\mu) D(\lambda)^{-1}$ from our normalization of $\phi$.

Inserting (4.1) into $D(\lambda)=1+\left\langle(x-\lambda)^{-1}, \bar{\psi} \phi\right\rangle$, we get a second equation relating $D(\lambda)$ and $D(\mu)$, allowing us to determine $D(\mu)$ and hence $\phi(x)$.

REMARK 4.4. Note that if $\operatorname{Ran} S_{\lambda, B}=\operatorname{Span}\left\{\frac{1}{x-\lambda}\right\}$ or $\operatorname{Ran} \widetilde{S}_{\mu, B^{*}}=$ $\operatorname{Span}\left\{\frac{1}{x-\mu}\right\}$, it is clear from (3.14) and (3.16) that the $M$-function in general does not contain sufficient information to recover $\phi$ and $\psi$.

The following shows that there is nothing like the result of Proposition 4.3 for Hain-Lüst operators, and therefore no similar result can hold in the abstract setting.

Proposition 4.5. Assume we know $\operatorname{Ran} S_{\lambda, B}$ and $\operatorname{Ran} \widetilde{S}_{\mu, B^{*}}$ for some $\lambda, \mu$ for the Hain-Lüst operator $\widetilde{A}^{*}$ in (3.2). Then $\widetilde{A}^{*}$ is not uniquely determined.

Proof. It is sufficient to show the claim for the case when the coefficients of the operator are real. Knowing the ranges of the solution operators corresponds to knowing the kernels of the maximal operators, and hence two 
linearly independent solutions to each of the following equations:

$$
\begin{aligned}
& \left(\begin{array}{cc}
-\frac{d^{2}}{d x^{2}}+q(x) & w(x) \\
\widetilde{w}(x) & u(x)
\end{array}\right)\left(\begin{array}{l}
y_{1} \\
z_{1}
\end{array}\right)=\lambda\left(\begin{array}{l}
y_{1} \\
z_{1}
\end{array}\right), \\
& \left(\begin{array}{cc}
-\frac{d^{2}}{d x^{2}}+q(x) & \widetilde{w}(x) \\
w(x) & u(x)
\end{array}\right)\left(\begin{array}{l}
y_{2} \\
z_{2}
\end{array}\right)=\mu\left(\begin{array}{l}
y_{2} \\
z_{2}
\end{array}\right) .
\end{aligned}
$$

Write the pairs of solutions as $\left(y_{1}, z_{1}\right),\left(\widehat{y}_{1}, \widehat{z}_{1}\right)$, and $\left(y_{2}, z_{2}\right),\left(\widehat{y}_{2}, \widehat{z}_{2}\right)$, respectively. Since

$$
z_{1}=\frac{\widetilde{w} y_{1}}{u-\lambda}, \quad \widehat{z}_{1}=\frac{\widetilde{w} \widehat{y}_{1}}{u-\lambda}, \quad z_{2}=\frac{w y_{2}}{u-\mu}, \quad \widehat{z}_{2}=\frac{w \widehat{y}_{2}}{u-\mu},
$$

setting $\alpha=\frac{\widetilde{w}}{u-\lambda}$ and $\beta=\frac{w}{u-\mu}$, we can write this as a linear system for $q, w$, $\tilde{w}$ and $u$. A calculation shows that the corresponding matrix does not have full rank for any $\alpha$ and $\beta$, so the system is not uniquely solvable.

4.2. Reconstruction from two bordered resolvents. By allowing ourselves information from two bordered resolvents belonging to different boundary conditions, we obtain reconstruction procedures for the $M$-function.

TheOrem 4.6. Assume $\left.P_{\widetilde{\mathcal{S}}}\left(A_{B}-\lambda\right)^{-1}\right|_{\overline{\mathcal{S}}}$ and $\left.P_{\widetilde{\mathcal{S}}}\left(A_{C}-\lambda\right)^{-1}\right|_{\overline{\mathcal{S}}}$ are known. In addition, assume that

(i) $\Gamma_{2}\left(A_{C}-\lambda\right)^{-1} \overline{\mathcal{S}}$ and $\widetilde{\Gamma}_{2}\left(A_{C}-\lambda\right)^{-*} \overline{\widetilde{\mathcal{S}}}$ are known,

(ii) $\Gamma_{2}\left(A_{C}-\lambda\right)^{-1} \overline{\mathcal{S}}$ is dense in $\mathcal{H}$, and $\widetilde{\Gamma}_{2}\left(A_{C}-\lambda\right)^{-*} \overline{\widetilde{\mathcal{S}}}$ is dense in $\mathcal{K}$,

(iii) $\operatorname{Ran}(B-C)$ is dense in $\mathcal{H}$, and $\operatorname{ker}(B-C)=\{0\}$.

Then $M_{B}(\lambda)$ can be recovered.

Proof. Let $\lambda \in \rho\left(A_{B}\right) \cap \rho\left(A_{C}\right)$. Then the Krein formula (2.6) gives $\left(A_{B}-\lambda\right)^{-1}-\left(A_{C}-\lambda\right)^{-1}=S_{\lambda, C}\left(I+(B-C) M_{B}(\lambda)\right)(B-C) \Gamma_{2}\left(A_{C}-\lambda\right)^{-1}$.

Now let $f \in \overline{\widetilde{\mathcal{S}}}$ and $g \in \overline{\mathcal{S}}$. Then we know

$$
\left\langle f,\left(A_{B}-\lambda\right)^{-1} g\right\rangle-\left\langle f,\left(A_{C}-\lambda\right)^{-1} g\right\rangle .
$$

Using (2.6), we obtain

$$
\begin{aligned}
\langle f, & \left.\left(A_{B}-\lambda\right)^{-1} g\right\rangle-\left\langle f,\left(A_{C}-\lambda\right)^{-1} g\right\rangle \\
= & \left\langle f, S_{\lambda, C}\left(I+(B-C) M_{B}(\lambda)\right)(B-C) \Gamma_{2}\left(A_{C}-\lambda\right)^{-1} g\right\rangle \\
= & \left\langle\widetilde{\Gamma}_{2}\left(A_{C}-\lambda\right)^{-*} f,\left(I+(B-C) M_{B}(\lambda)\right)(B-C) \Gamma_{2}\left(A_{C}-\lambda\right)^{-1} g\right\rangle \\
= & \left\langle\widetilde{\Gamma}_{2}\left(A_{C}-\lambda\right)^{-*} f,(B-C) \Gamma_{2}\left(A_{C}-\lambda\right)^{-1} g\right\rangle \\
& +\left\langle(B-C)^{*} \widetilde{\Gamma}_{2}\left(A_{C}-\lambda\right)^{-*} f, M_{B}(\lambda)(B-C) \Gamma_{2}\left(A_{C}-\lambda\right)^{-1} g\right\rangle .
\end{aligned}
$$

Our assumptions now allow us to recover $M_{B}(\lambda)$. 
REMARK 4.7. Alternatively, knowing the projection to $\overline{\widetilde{\mathcal{S}}}$ of the derivative of the resolvent with respect to the boundary condition and one resolvent restricted to $\overline{\mathcal{S}}$ will suffice, because for $C=B+\varepsilon D$ we deduce from (2.6) that

$$
\frac{\left(A_{B}-\lambda\right)^{-1}-\left(A_{C}-\lambda\right)^{-1}}{\varepsilon} \rightarrow S_{\lambda, B} D \Gamma_{2}\left(A_{B}-\lambda\right)^{-1} \quad \text { as } \varepsilon \rightarrow 0 .
$$

If we now have the assumption of density of $D \Gamma_{2}\left(A_{B}-\lambda\right)^{-1} \overline{\mathcal{S}}$, then for $f \in \overline{\mathcal{S}}$ and $g \in \overline{\widetilde{\mathcal{S}}}$, since

$$
\left\langle S_{\lambda, B} D \Gamma_{2}\left(A_{B}-\lambda\right)^{-1} f, g\right\rangle=\left\langle D \Gamma_{2}\left(A_{B}-\lambda\right)^{-1} f, \widetilde{\Gamma}_{2}\left(A_{B}-\lambda\right)^{-*} g\right\rangle,
$$

and $\left\langle S_{\lambda, B} D \Gamma_{2}\left(A_{B}-\lambda\right)^{-1} f, g\right\rangle$ is known from the derivative, while $D \Gamma_{2}\left(A_{B}-\lambda\right)^{-1} f$ is known from the restricted resolvent, knowing the projection of the derivative to $\overline{\widetilde{\mathcal{S}}}$ corresponds to knowing $\left.\widetilde{\Gamma}_{2}\left(A_{B}-\lambda\right)^{-*}\right|_{\widetilde{\mathcal{S}}}$.

4.3. Information on the resolvent from the $M$-function. The following result gives some insight into the problem of reconstructing $A_{B}$ from $M_{B}(\lambda)$. From some examples given later, we will see that the knowledge of $M_{B}(\lambda)$ does not allow the reconstruction of the bordered resolvent.

Theorem 4.8. Assume we know $M_{B}(\lambda)$ for all $\lambda \in \rho\left(A_{B}\right)$, $\operatorname{Ran} S_{\mu, B}$ for all $\mu \in \Lambda$, and $\operatorname{Ran} \widetilde{S}_{\widetilde{\mu}, B^{*}}$ for all $\widetilde{\mu} \in \widetilde{\Lambda}$, where $\Lambda \subseteq \rho\left(A_{B}\right)$ and $\widetilde{\Lambda} \subseteq \rho\left(A_{B}^{*}\right)$ are dense subsets. Then we can find $P_{\widetilde{\mathcal{S}}}\left(A_{B}-\lambda\right)^{-1} P_{\overline{\mathcal{S}}}$ for all $\lambda \in \rho\left(A_{B}\right)$.

Proof. Let $\mu \in \Lambda$ and $\widetilde{\mu} \in \widetilde{\Lambda}$. Consider (2.7) for any $F \in \operatorname{Ran} S_{\mu, B}$ and $v \in \operatorname{Ran} \widetilde{S}_{\widetilde{\mu}, B^{*}}$. Then

$$
\begin{aligned}
\langle F & \left.-\left(A_{B}-\lambda\right)^{-1}\left(\widetilde{A}^{*}-\lambda\right) F,\left(A^{*}-\bar{\lambda} I\right) v\right\rangle \\
& =\left\langle F-\left(A_{B}-\lambda\right)^{-1}(\mu-\lambda) F,(\widetilde{\mu}-\bar{\lambda} I) v\right\rangle \\
& =-\left\langle\left(\Gamma_{1}-B \Gamma_{2}\right) F, \widetilde{\Gamma}_{2} v\right\rangle_{\mathcal{H}}+\left\langle M_{B}(\lambda)\left(\Gamma_{1}-B \Gamma_{2}\right) F,\left(\widetilde{\Gamma}_{1}-B^{*} \widetilde{\Gamma}_{2}\right) v\right\rangle_{\mathcal{K}} .
\end{aligned}
$$

We know the r.h.s. of this equation for any $F \in \operatorname{Ran} S_{\mu, B}, v \in \operatorname{Ran} \widetilde{S}_{\widetilde{\mu}, B^{*}}$ and $\lambda \in \rho\left(A_{B}\right)$, so we know

$$
\left\langle F-\left(A_{B}-\lambda\right)^{-1}(\mu-\lambda) F,(\widetilde{\mu}-\bar{\lambda} I) v\right\rangle .
$$

Choosing $\lambda \neq \mu$ and $\lambda \neq \overline{\widetilde{\mu}}$, we know

$$
\left\langle\left(A_{B}-\lambda\right)^{-1} F, v\right\rangle=\left\langle P_{\widetilde{\mathcal{S}}}\left(A_{B}-\lambda\right)^{-1} P_{\overline{\mathcal{S}}} F, v\right\rangle
$$

for any $F \in \operatorname{Ran} S_{\mu, B}, v \in \operatorname{Ran} \widetilde{S}_{\widetilde{\mu}, B^{*}}$ and $\lambda \in \rho\left(A_{B}\right) \backslash(\{\mu\} \cup\{\overline{\widetilde{\mu}}\})$. By continuity, we know it for all $\lambda \in \rho\left(A_{B}\right)$.

As $\operatorname{Span}\left\{\operatorname{Ran} S_{\mu, B}: \mu \in \Lambda\right\}$ is dense in $\overline{\mathcal{S}}$ and $\operatorname{Span}\left\{\operatorname{Ran} \widetilde{S}_{\widetilde{\mu}, B^{*}}: \widetilde{\mu} \in \widetilde{\Lambda}\right\}$ is dense in $\overline{\widetilde{\mathcal{S}}}$, the boundedness of $P_{\widetilde{\mathcal{S}}}\left(A_{B}-\lambda\right)^{-1} P_{\overline{\mathcal{S}}}$ gives the result. 
5. Analytic continuation. We now discuss the relationship between the analytic continuation of $M_{B}(\lambda)$ and the analytic continuation of the bordered resolvent of $A_{B}$, both initially defined on the resolvent set of $A_{B}$.

Theorem 5.1. Let $\mu, \overline{\widetilde{\mu}} \in \rho\left(A_{B}\right)$. Assume that for any $F \in \operatorname{Ran} S_{\mu, B}$ and $v \in \operatorname{Ran} \widetilde{S}_{\widetilde{\mu}, B^{*}}$,

$$
\left\langle\left.\left(A_{B}-\lambda\right)^{-1}\right|_{\overline{\mathcal{S}}} F, v\right\rangle
$$

admits an analytic continuation to some domain $D$ of the complex plane (possibly on a different Riemann sheet). Then $M_{B}(\cdot)$ admits an analytic continuation to the same domain D.

Proof. Given $f \in \mathcal{H}$ and $\widetilde{f} \in \mathcal{K}$, choose $F=S_{\mu, B} f$ and $v=\widetilde{S}_{\widetilde{\mu}, B^{*}} \widetilde{f}$. Then (2.7) becomes

$$
\left\langle F-(\mu-\lambda)\left(A_{B}-\lambda\right)^{-1} F,(\widetilde{\mu}-\bar{\lambda}) v\right\rangle=-\left\langle f, \widetilde{\Gamma}_{2} v\right\rangle_{\mathcal{H}}+\left\langle M_{B}(\lambda) f, \widetilde{f}\right\rangle_{\mathcal{K}},
$$

and the 1.h.s. admits an analytic continuation, so the r.h.s. does as well.

Lemma 5.2. For $\mu \in \rho\left(A_{B}\right)$,

$$
\left(\frac{d}{d \lambda} S_{\cdot, B}\right)(\mu)=\left(A_{B}-\mu\right)^{-1} S_{\mu, B} .
$$

Proof. From Lemma 2.5, we have

$$
\frac{S_{\lambda, B} f-S_{\mu, B} f}{\lambda-\mu}=\left(A_{B}-\lambda\right)^{-1} S_{\mu, B} f,
$$

which immediately proves the assertion.

The next result is a generalization of [16, Theorem 3.5].

TheOREM 5.3. Assume $M_{B}(\cdot)$ admits an analytic continuation to some domain $D$ of the complex plane (possibly on a different Riemann sheet). Let $\mu, \overline{\widetilde{\mu}} \in \rho\left(A_{B}\right)$. Then $\left\langle\left.\left(A_{B}-\lambda\right)^{-1}\right|_{\mathcal{S}} F, v\right\rangle$ admits an analytic continuation to the same domain $D$ for any $F \in \operatorname{Ran} S_{\mu, B}$ and $v \in \operatorname{Ran} \widetilde{S}_{\widetilde{\mu}, B^{*}}$, apart from possible simple poles at $\mu$ and $\overline{\widetilde{\mu}}$. If $\mu=\overline{\widetilde{\mu}}$, a pole of order 2 is possible at this point.

Proof. Let $F \in \operatorname{Ran} S_{\mu, B}$ and $v \in \operatorname{Ran} \widetilde{S}_{\widetilde{\mu}, B^{*}}$. By assumption the r.h.s. of (2.7) admits an analytic continuation, hence so does the l.h.s., which is given by $\left\langle F-(\mu-\lambda)\left(A_{B}-\lambda\right)^{-1} F,(\widetilde{\mu}-\bar{\lambda}) v\right\rangle$. Since $\langle F,(\widetilde{\mu}-\bar{\lambda}) v\rangle$ is clearly analytic, we see that $(\mu-\lambda)(\overline{\widetilde{\mu}}-\lambda)\left\langle\left(A_{B}-\lambda\right)^{-1} F, v\right\rangle$ is analytic, which gives the desired result.

REMARK 5.4. We can extend the set of those vectors for which

$$
\left\langle\left.\left(A_{B}-\lambda\right)^{-1}\right|_{\mathcal{S}} F, v\right\rangle
$$

admits an analytic continuation by developing vectors on both sides by taking linear combinations and using the resolvents of $A_{B}$ and $\widetilde{A}_{B^{*}}$ respectively. 
However, we should not expect the result to extend to the whole of $\overline{\mathcal{S}}$ (or $\overline{\widetilde{\mathcal{S}}}$ ), and therefore the bordered resolvent will not necessarily admit an analytic continuation.

It is interesting to note that poles of $\left\langle\left.\left(A_{B}-\lambda\right)^{-1}\right|_{\mathcal{S}} F, v\right\rangle$ at $\mu$ and $\overline{\widetilde{\mu}}$ do arise in concrete examples, though they may sometimes be cancelled by other terms.

ExAmple 5.5. Let $H_{2}^{ \pm}$denote the Hardy classes (see [30]). Let $\mu \in \mathbb{C}^{-}$ and $\widetilde{\mu} \in \mathbb{C}^{+}$. Consider an example of the Friedrichs model from Section 3.3, where $\phi \in H_{2}^{-}$and $\psi \in H_{2}^{+}$are rational functions with poles in suitable half-planes such that $\psi(\lambda) \overline{\phi(\bar{\lambda})}$ does not have poles at $\mu$ or $\overline{\widetilde{\mu}}$. Then let

$$
F_{\mu}:=\frac{1}{x-\mu} \in H_{2}^{+} \cap \operatorname{ker}\left(\widetilde{A}^{*}-\mu\right), \quad v_{\widetilde{\mu}}:=\frac{1}{x-\widetilde{\mu}} \in H_{2}^{-} \cap \operatorname{ker}\left(A^{*}-\widetilde{\mu}\right) .
$$

We consider the analytic continuation of the functions $\left\langle\left(A_{B}-\cdot\right)^{-1} F_{\mu}, v_{\widetilde{\mu}}\right\rangle$ and $M_{B}(\cdot)$ from the upper to the lower half-plane. From (2.7), for $\lambda \in \mathbb{C}^{+}$ we get

$$
\begin{aligned}
(\lambda-\mu)(\lambda-\overline{\widetilde{\mu}})\left\langle\left( A_{B}\right.\right. & \left.-\lambda)^{-1} F_{\mu}, v_{\widetilde{\mu}}\right\rangle \\
= & -\left\langle M_{B}(\lambda)\left(\Gamma_{1}-B \Gamma_{2}\right) F_{\mu},\left(\widetilde{\Gamma}_{1}-B^{*} \widetilde{\Gamma}_{2}\right) v_{\widetilde{\mu}}\right\rangle \\
& \left.+\left\langle\left(\Gamma_{1}-B \Gamma_{2}\right) F_{\mu}, \widetilde{\Gamma}_{2} v_{\widetilde{\mu}}\right)\right\rangle+\left\langle F_{\mu},(\widetilde{\mu}-\bar{\lambda}) v_{\widetilde{\mu}}\right\rangle .
\end{aligned}
$$

Now, $\left(\widetilde{\Gamma}_{1}-B^{*} \widetilde{\Gamma}_{2}\right) v_{\widetilde{\mu}}=\pi i-B^{*}, \widetilde{\Gamma}_{2} v_{\mu}=1$, and

$$
\begin{aligned}
\left\langle F_{\mu}, v_{\widetilde{\mu}}\right\rangle & =\left\langle\frac{1}{x-\mu}, \frac{1}{x-\widetilde{\mu}}\right\rangle=0, \\
\left(\Gamma_{1}-B \Gamma_{2}\right) F_{\mu} & =\left(\Gamma_{1}-B \Gamma_{2}\right) \frac{1}{x-\mu}=-\pi i-B .
\end{aligned}
$$

Thus (5.1) gives

$$
\begin{aligned}
(\lambda-\mu)(\lambda-\overline{\widetilde{\mu}})\left\langle\left(A_{B}-\lambda\right)^{-1} F_{\mu}, v_{\widetilde{\mu}}\right\rangle & =-M_{B}(\lambda)(\pi i+B)(\pi i+B)-\pi i-B \\
& =-(\pi i+B)\left(M_{B}(\lambda)(\pi i+B)+1\right),
\end{aligned}
$$

or

$$
\begin{aligned}
\left\langle\left(A_{B}-\lambda\right)^{-1} F_{\mu}, v_{\widetilde{\mu}}\right\rangle & =-\frac{(\pi i+B)\left(M_{B}(\lambda)(\pi i+B)+1\right)}{(\lambda-\mu)(\lambda-\overline{\widetilde{\mu}})} \\
& =\frac{-2 \pi i\left[\frac{\pi i-B}{\pi i+B}-2 \pi i \psi(\lambda) \overline{\phi(\bar{\lambda})}\right]^{-1}}{(\lambda-\mu)(\lambda-\overline{\widetilde{\mu}})} .
\end{aligned}
$$


From (3.16) we get

$$
\begin{aligned}
M_{B}(\lambda) & =\left[\pi i+\frac{4 \pi^{2} \psi(\lambda) \overline{\phi(\bar{\lambda})}}{1+2 \pi i \psi(\lambda) \overline{\phi(\bar{\lambda})}}-B\right]^{-1} \\
& =\left[-\pi i-B+\frac{2 \pi i}{1+2 \pi i \psi(\lambda) \overline{\phi(\bar{\lambda})}}\right]^{-1} .
\end{aligned}
$$

Thus the function $M_{B}(\cdot)$ admits an analytic continuation to the lower halfplane, while the analytic continuation of $\left\langle\left(A_{B}-\cdot\right)^{-1} F_{\mu}, v_{\tilde{\mu}}\right\rangle$ given by $(5.2)$ has poles at $\mu$ and $\overline{\widetilde{\mu}}$.

In the case when $B \neq-\pi i$, to cancel the poles in (5.2) we need to choose poles of the analytic continuation of $\psi(\lambda) \overline{\phi(\bar{\lambda})}$ to lie at $\mu$ and $\overline{\widetilde{\mu}}$. Note: the poles appearing in Theorem 5.3 should not be confused with resonances (poles of the analytic continuation of $M_{B}$ ). Here the resonances are due to zeroes of $\frac{\pi i-B}{\pi i+B}-2 \pi i \psi(\lambda) \overline{\phi(\bar{\lambda})}$ in $(5.2)$.

6. Abstract theory: relation between jumps of $M_{B}$ and bordered resolvent. We consider the case in which $A_{B}$ and $A_{B}^{*}$ have essential spectrum lying on the real axis; we wish to examine in what sense $M_{B}(\lambda)$ jumps across the real axis, and how this may be related to a jump in the resolvent $\left(A_{B}-\lambda\right)^{-1}$. We started investigating this question in [17, Section 5], where we showed that under certain hypotheses existence of a jump for the resolvent is equivalent to existence of a jump for the $M$-function. Here we go much further, investigating the rank of the jump (corresponding to the multiplicity of the a.c. spectrum) under relaxed assumptions. This is an important step in the development of a scattering theory for the kinds of operators under consideration.

Assumption 1. We assume that there exist countable families $\left\{f_{i}\right\}_{i \in I}$ and $\left\{w_{j}\right\}_{j \in \tilde{I}}$ in $\mathcal{H}$ and $\mathcal{K}$ respectively, whose closed linear spans are $\mathcal{H}$ and $\mathcal{K}$, and that for these families the inner products $\left\langle M_{B}(\lambda) f_{i}, w_{j}\right\rangle$ lie in both the Nevanlinna classes $N\left(\mathbb{C}_{ \pm}\right)$. This implies that they have non-tangential boundary values $\left\langle M_{B}(k \pm i 0) f_{i}, w_{j}\right\rangle$ for a.e. $k \in \mathbb{R}$. The class $N\left(\mathbb{C}_{ \pm}\right)$consists of all meromorphic functions on $\mathbb{C}_{ \pm}$which can be represented as the quotient of two bounded analytic functions in the corresponding half-plane (see [31]).

Our first result is that this assumption is equivalent to an assumption on the resolvent.

Lemma 6.1. The functions $\left\langle M_{B}(\lambda) f_{i}, w_{j}\right\rangle$ lie in $N\left(\mathbb{C}_{ \pm}\right)$if and only if, for all $\mu \notin \sigma\left(A_{B}\right)$ and $\widetilde{\mu} \notin \sigma\left(\widetilde{A}_{B^{*}}\right)$, the functions $\left\langle\left(A_{B}-\lambda\right)^{-1} S_{\mu, B} f_{i}, \widetilde{S}_{\widetilde{\mu}, B^{*}} w_{j}\right\rangle$ lie in $N\left(\mathbb{C}_{ \pm}\right)$. 
Proof. Starting with the fundamental identity

$$
\left\langle\left(\widetilde{A}^{*}-\lambda\right) u, v\right\rangle-\left\langle u,\left(A^{*}-\bar{\lambda}\right) v\right\rangle=\left\langle\Gamma_{1} u, \widetilde{\Gamma}_{2} v\right\rangle-\left\langle\Gamma_{2} u, \widetilde{\Gamma}_{1} v\right\rangle
$$

and making the choices $u=\left(A_{B}-\lambda\right)^{-1} S_{\mu, B} f_{i}, v=\widetilde{S}_{\widetilde{\mu}, B^{*}} w_{j}$ leads to

$$
\begin{aligned}
& \quad\left\langle S_{\mu, B} f_{i}, w_{j}\right\rangle-\left\langle\left(A_{B}-\lambda\right)^{-1} S_{\mu, B} f_{i},(\widetilde{\mu}-\bar{\lambda}) \widetilde{S}_{\widetilde{\mu}, B^{*}} w_{j}\right\rangle \\
& =\left\langle\Gamma_{2}\left(A_{B}-\lambda\right)^{-1} S_{\mu, B} f_{i}, \widetilde{\Gamma}_{1} \widetilde{S}_{\widetilde{\mu}, B^{*}} w_{j}\right\rangle-\left\langle\Gamma_{2}\left(A_{B}-\lambda\right)^{-1} S_{\mu, B} f_{i}, \widetilde{\Gamma}_{1} \widetilde{S}_{\widetilde{\mu}, B^{*}} w_{j}\right\rangle .
\end{aligned}
$$

If the functions $\left\langle M_{B}(\lambda) f_{i}, w_{j}\right\rangle$ lie in $N\left(\mathbb{C}_{ \pm}\right)$then, thanks to the identity

$$
M_{B}(\lambda)=\Gamma_{2}\left(I+(\lambda-\mu)\left(A_{B}-\lambda\right)^{-1}\right) S_{\mu, B},
$$

the terms $\left\langle\Gamma_{2}\left(A_{B}-\lambda\right)^{-1} S_{\mu, B} f_{i}, \cdot\right\rangle$ appearing in (6.1) also lie in $N\left(\mathbb{C}_{ \pm}\right)$, so

$$
\left\langle\left(A_{B}-\lambda\right)^{-1} S_{\mu, B} f_{i},(\widetilde{\mu}-\bar{\lambda}) \widetilde{S}_{\widetilde{\mu}, B^{*}} w_{j}\right\rangle
$$

lies in $N\left(\mathbb{C}_{ \pm}\right)$. This implies that $\left\langle\left(A_{B}-\lambda\right)^{-1} S_{\mu, B} f_{i}, \widetilde{S}_{\widetilde{\mu}, B^{*}} w_{j}\right\rangle$ lies in $N\left(\mathbb{C}_{ \pm}\right)$.

The converse result is immediate from equation (6.2): if inner products of the form

$$
\left\langle\left(A_{B}-\lambda\right)^{-1} S_{\mu, B} f_{i}, \widetilde{S}_{\widetilde{\mu}, B^{*}} w_{j}\right\rangle
$$

lie in $N\left(\mathbb{C}_{ \pm}\right)$then so do the inner products $\left\langle M_{B}(\lambda) f_{i}, w_{j}\right\rangle$.

TheORem 6.2. Suppose that Assumption 1 holds and

$$
\varepsilon\left|\left\langle M_{B}(k \pm i \varepsilon) f, w\right\rangle\right| \rightarrow 0 \quad \text { as } \varepsilon \searrow 0
$$

for a.e. $k \in \mathbb{R}$ and for $f, w$ in a dense countable subset of the boundary spaces. Choose sets $\left\{\mu_{i}\right\}_{i \in I},\left\{\widetilde{\mu}_{j}\right\}_{j \in \tilde{I}}$ non-real and outside $\sigma\left(A_{B}\right)$. Let $F_{i}=$ $S_{\mu_{i}, B} f_{i}$ and $v_{j}=\widetilde{S}_{\widetilde{\mu}_{j}, B^{*}} w_{j}$. Then for a.e. $k \in \mathbb{R}$,

$$
\operatorname{rank}\left(\left[P_{\left\{v_{j}\right\}}\left(A_{B}-\lambda\right)^{-1} P_{\left\{F_{i}\right\}}\right]_{\lambda=k}\right)=\operatorname{rank}\left(\left[P_{\left\{w_{j}\right\}} M_{B}(\lambda) P_{\left\{f_{i}\right\}}\right]_{\lambda=k}\right),
$$

where $P_{\left\{v_{j}\right\}}$ and $P_{\left\{w_{j}\right\}}$ denote the projections onto the indicated one-dimensional spaces, and $[\cdot]_{\lambda=k}$ denotes the jump between $\lambda=k+i \varepsilon$ and $\lambda=k-i \varepsilon$ as $\varepsilon \searrow 0$.

In order to prove Theorem 6.2 we require the following lemma.

Lemma 6.3. The collections $\left\{F_{i}\right\}_{i \in I}$ and $\left\{v_{j}\right\}_{j \in \tilde{I}}$ from Theorem 6.2 are both linearly independent.

Proof. We give the proof for $\left\{F_{i}\right\}_{i \in I}$; the remaining case is similar. Assume that there are some constants $\alpha_{i}$ such that $\sum_{i} \alpha_{i} F_{i}=0$. Let $\zeta \in \mathbb{C}$; applying $\left(\widetilde{A}^{*}-\zeta\right)^{k}$ for some $k \in \mathbb{N}$ we get $\sum_{i} \alpha_{i}\left(\mu_{i}-\zeta\right)^{k} F_{i}=0$. First, assume all the $\mu_{i}$ are distinct. Then we can choose $i_{0}$ and $\zeta$ such that $\left|\mu_{i_{0}}-\zeta\right|>\left|\mu_{i}-\zeta\right|$ for $i \neq i_{0}$. Letting $k \rightarrow \infty$ we deduce that $\alpha_{i_{0}} F_{i_{0}}=0$. Proceeding in this way we get $\alpha_{i}=0$ for all $i$ as long as the $\mu_{i}$ are distinct.

If we have a collection of $\mu_{i}$ which are all equal, say for $i \in J$, where $J$ is some index set, then we can prove that for some appropriately chosen $\zeta$ we have $0=\sum_{i \in J} \alpha_{i}\left(\mu_{i}-\zeta\right)^{k} S_{\mu_{i}, B} f_{i}$, giving $S_{\mu_{\ell}, B} \sum_{i \in J} \alpha_{i} f_{i}=0$ for $\ell \in J$. 
This implies that $\sum_{i \in J} \alpha_{i} f_{i}=0$, and hence $\alpha_{i}=0$ for all $i \in J$, by linear independence of the $\left\{f_{i}\right\}$.

COROLlary 6.4. The collections $\left\{\left(\mu_{i}-k\right) F_{i}\right\}$ and $\left\{\left(\overline{\widetilde{\mu}}_{j}-k\right) v_{j}\right\}$ are both linearly independent as long as $\mu_{i} \neq k$ and $\overline{\widetilde{\mu}}_{j} \neq k$ for all $i, j$.

Proof of Theorem 6.2. We use the fundamental identity (2.7), which yields

$$
\begin{aligned}
\left\langle F_{i}-\left(A_{B}-\lambda\right)^{-1}\left(\widetilde{A}^{*}-\lambda\right) F_{i},\right. & \left.\left(A^{*}-\bar{\lambda} I\right) v_{j}\right\rangle \\
& =-\left\langle f_{i}, \widetilde{\Gamma}_{2} v_{j}\right\rangle_{\mathcal{H}}+\left\langle M_{B}(\lambda) f_{i}, w_{j}\right\rangle_{\mathcal{K}}
\end{aligned}
$$

for all $i \in I, j \in \tilde{I}$ and $\lambda=k+i \varepsilon$. The jump at $k$ of the right hand side is clearly given by $\left[\left\langle M_{B}(k) f_{i}, w_{j}\right\rangle_{\mathcal{K}}\right]$, which for convenience we denote by $\left\langle\left[M_{B}\right](k) f_{i}, w_{j}\right\rangle_{\mathcal{K}}$. By our assumptions on the $\left\{f_{i}\right\}$ and $\left\{w_{j}\right\}$, this is non-zero if and only if $\left[M_{B}\right](k) \neq 0$.

Now consider the left hand side of (6.4). Clearly, $\left\langle F_{i},\left(A^{*}-\bar{\lambda} I\right) v_{j}\right\rangle$ has no jump. For

$$
G(\lambda):=\left\langle\left(A_{B}-\lambda\right)^{-1} F_{i}, A^{*} v_{j}\right\rangle+\left\langle\left(A_{B}-\lambda\right)^{-1} \widetilde{A}^{*} F_{i}, v_{j}\right\rangle
$$

the negative of the remaining term on the left hand side of (6.4) is

$$
\begin{aligned}
& \left\langle\left(A_{B}-\lambda\right)^{-1}\left(\widetilde{A}^{*}-\lambda\right) F_{i},\left(A^{*}-\bar{\lambda}\right) v_{j}\right\rangle \\
& \quad=\left\langle\left(A_{B}-\lambda\right)^{-1} \widetilde{A}^{*} F_{i}, A^{*} v_{j}\right\rangle-\lambda G(\lambda)+|\lambda|^{2}\left\langle\left(A_{B}-\lambda\right)^{-1} F_{i}, v_{j}\right\rangle .
\end{aligned}
$$

Observe that $[\lambda G(\lambda)]=k[G](k)+\lim _{\varepsilon \searrow 0} i \varepsilon(G(k+i \varepsilon)+G(k-i \varepsilon))$. We shall prove later that

$$
\lim _{\varepsilon \searrow 0} \varepsilon\left\langle\left(A_{B}-\lambda\right)^{-1} F_{i}, v_{j}\right\rangle=0 \quad \text { for a.e. } k .
$$

This implies $[\lambda G(\lambda)]=k[G](k)$ and

$$
\left[|\lambda|^{2}\left\langle\left(A_{B}-\lambda\right)^{-1} F_{i}, v_{j}\right\rangle\right]=k^{2}\left\langle\left[\left(A_{B}-\lambda\right)^{-1}\right] F_{i}, v_{j}\right\rangle ;
$$

hence, from (6.6), the formula

$$
-\left[\left\langle\left(A_{B}-\lambda\right)^{-1}\left(\widetilde{A}^{*}-\lambda\right) F_{i},\left(A^{*}-\bar{\lambda}\right) v_{j}\right\rangle\right]=\left\langle\left[\left(A_{B}-\lambda\right)^{-1}\right]\left(\widetilde{A}^{*}-k\right) F_{i},\left(A^{*}-k\right) v_{j}\right\rangle
$$

gives the jump of the left hand side of (6.4). Therefore

$$
-\left(\mu_{i}-k\right)\left(\overline{\widetilde{\mu}}_{j}-k\right)\left\langle\left[\left(A_{B}-\lambda\right)^{-1}\right] F_{i}, v_{j}\right\rangle=\left\langle\left[M_{B}\right](k) f_{i}, w_{j}\right\rangle_{\mathcal{K}},
$$

from which our result follows.

It remains to establish (6.7). Returning to (6.4) we have

$$
\begin{aligned}
-\left(\mu_{i}-\lambda\right)\left(\overline{\widetilde{\mu}}_{j}-\lambda\right) & \left\langle\left(A_{B}-\lambda\right)^{-1} F_{i}, v_{j}\right\rangle \\
= & \left(\overline{\widetilde{\mu}}_{j}-\lambda\right)\left\langle F_{i}, v_{j}\right\rangle-\left\langle f_{i}, \widetilde{\Gamma}_{2} v_{j}\right\rangle_{\mathcal{H}}+\left\langle M_{B}(\lambda) f_{i}, w_{j}\right\rangle_{\mathcal{K}},
\end{aligned}
$$


whence

$$
\left\langle\left(A_{B}-\lambda\right)^{-1} F_{i}, v_{j}\right\rangle \mid \leq \frac{\left|\overline{\widetilde{\mu}}_{j}-\lambda\right|\left|\left\langle F_{i}, v_{j}\right\rangle\right|+\left|\left\langle f_{i}, \widetilde{\Gamma}_{2} v_{j}\right\rangle_{\mathcal{H}}\right|+\left|\left\langle M_{B}(\lambda) f_{i}, w_{j}\right\rangle \mathcal{K}\right|}{\left|\mu_{i}-\lambda\right|\left|\widetilde{\widetilde{\mu}}_{j}-\lambda\right|} .
$$

Thus (6.7) is an immediate consequence of the hypothesis that (6.3) holds for a.e. $k \in \mathbb{R}$.

ThEOREM 6.5. Let $\left\{f_{i}\right\}_{i \in I}$ and $\left\{w_{j}\right\}_{j \in \tilde{I}}$ be linearly independent vectors whose spans are dense in $\mathcal{H}$ and $\mathcal{K}$ respectively. Let $\left\{\mu_{\ell}\right\}_{\ell \in J}$ and $\left\{\widetilde{\mu}_{\nu}\right\}_{\nu \in \tilde{J}}$ be collections of distinct strictly complex numbers dense in $\mathbb{C} \backslash \sigma\left(A_{B}\right)$ and $\mathbb{C} \backslash \sigma\left(A_{B}^{*}\right)$ respectively. Define $F_{i, \ell}=S_{\mu_{\ell}, B} f_{i}, v_{j, \nu}=\widetilde{S}_{\widetilde{\mu}_{\nu}, B^{*}} w_{j}$. Then:

(i) The collections $\left\{F_{i, \ell}\right\}_{i \in I, \ell \in J}$ and $\left\{v_{j, \nu}\right\}_{j \in \tilde{I}, \nu \in \tilde{J}}$ are both linearly independent and their spans are dense in $\overline{\mathcal{S}}$ and $\overline{\widetilde{\mathcal{S}}}$ respectively.

(ii) For $N, M \in \mathbb{N}$ let $P_{N, \overline{\mathcal{S}}}$ and $P_{M, \overline{\mathcal{S}}}$ denote projections onto the $N$ - and $M$-dimensional subspaces of $\overline{\mathcal{S}}$ and $\overline{\widetilde{\mathcal{S}}}$, respectively, spanned by $N$ of the $F_{i, \ell}$ and $M$ of the $v_{j, \nu}$ respectively, chosen so that $\lim _{N \rightarrow \infty} P_{N, \overline{\mathcal{S}}}$ $=I$ and $\lim _{M \rightarrow \infty} P_{M, \overline{\mathcal{S}}}=I$, in the sense of strong convergence. Let

$$
\begin{aligned}
& E_{1}=\left\{k \in \mathbb{R}:\left[M_{B}\right](k) \text { exists in the weak topology }\right\} \\
& E_{2}=\left\{k \in \mathbb{R}: \lim _{\varepsilon \searrow 0} \varepsilon\left|\left\langle M_{B}(k \pm i \varepsilon) f_{i}, w_{j}\right\rangle\right|=0 \text { for all } i, j\right\} .
\end{aligned}
$$

Then $\left[P_{M, \overline{\widetilde{\mathcal{S}}}}\left(A_{B}-\lambda\right)^{-1} P_{N, \overline{\mathcal{S}}}\right](k)$ exists for any $k \in E_{1} \cap E_{2} ;$ moreover

$$
\lim _{N, M \rightarrow \infty} \operatorname{rank}\left(\left.\left[P_{M, \overline{\widetilde{\mathcal{S}}}}\left(A_{B}-\lambda\right)^{-1} P_{N, \overline{\mathcal{S}}}\right]\right|_{\lambda=k}\right)
$$

exists and is equal to $\operatorname{rank}\left(\left[M_{B}\right](k)\right)$.

Proof. (i) The fact that the closed linear spans of the sets $\left\{F_{i, \ell}\right\}_{i \in I, \ell \in J}$ and $\left\{v_{j, \nu}\right\}_{j \in \tilde{I}, \nu \in \tilde{J}}$ are $\overline{\mathcal{S}}$ and $\overline{\widetilde{\mathcal{S}}}$ follows immediately from the definitions of $\overline{\mathcal{S}}$ and $\overline{\mathcal{S}}$ together with the fact that the closed linear spans of $\left\{f_{i}\right\}_{i \in I}$ and $\left\{w_{j}\right\}_{j \in \tilde{I}}$ are $\mathcal{H}$ and $\mathcal{K}$ respectively. It only remains to establish linear independence. Assume that there exist constants $\alpha_{i, \ell}$ such that $\sum_{i, \ell} \alpha_{i, \ell} F_{i, \ell}=0$. This means that $\sum_{i, \ell} \alpha_{i, \ell} S_{\mu_{\ell}, B} f_{i}=0$. Applying $\widetilde{A}^{*} k$ times yields

$$
\sum_{i, \ell} \alpha_{i, \ell} \mu_{l}^{k} F_{i, \ell}=\sum_{\ell} \mu_{l}^{k} \sum_{i} \alpha_{i, \ell} F_{i, \ell}=0, \quad k=0,1,2, \ldots
$$

Since the $\mu_{l}$ are distinct this yields $\sum_{i} \alpha_{i, \ell} F_{i, \ell}=0$ for all $\ell$. This means $S_{\mu_{\ell}, B} \sum_{i} \alpha_{i, \ell} f_{i}=0$, and since $S_{\mu_{\ell}, B}$ has a left inverse this implies $\sum_{i} \alpha_{i, \ell} f_{i}$ $=0$. But the $\left\{f_{i}\right\}$ are linearly independent, so $\alpha_{i, \ell}=0$ for all $i$ and $\ell$.

(ii) Let $P_{N, \overline{\mathcal{S}}}$ denote the projection onto $N$ of the $F_{i, \ell}$, and $P_{M, \overline{\widetilde{\mathcal{S}}}}$ denote the projection onto $M$ of the $v_{j, \nu}$, chosen in each case to be such that $P_{N, \overline{\mathcal{S}}}$ 
and $P_{M, \overline{\widetilde{\mathcal{S}}}}$ converge strongly to the identity. Let $P_{N^{\prime}}$ and $\widetilde{P}_{M^{\prime}}$ denote the projections onto the spaces spanned by the corresponding $f_{i}$, of which there will be $N^{\prime} \leq N$, and $w_{j}$, of which there will be $M^{\prime} \leq M$. Since $k \in E_{1} \cap E_{2}$ we may invoke (6.8) from the proof of Theorem 6.2 and deduce that

$$
\begin{aligned}
-\left(\mu_{\ell}-k\right)\left(\widetilde{\widetilde{\mu}}_{\nu}-k\right)\left\langle\left[P_{M, \overline{\mathcal{S}}}\left(A_{B}-\lambda\right)^{-1} P_{N, \overline{\mathcal{S}}}\right]_{\lambda=k} F_{i, \ell}, v_{j, \nu}\right\rangle \\
=\left\langle\left[\widetilde{P}_{M^{\prime}} M_{B}(\lambda) P_{N^{\prime}}\right]_{\lambda=k} f_{i}, w_{j}\right\rangle .
\end{aligned}
$$

As $k \in \mathbb{R}$ we know that $\mu_{\ell} \neq k$ and $\widetilde{\mu}_{\nu} \neq k$, so we define $X_{i, \ell}=\left(\mu_{\ell}-k\right) F_{i, \ell}$ and $Y_{j, \nu}=-\left(\widetilde{\mu}_{\nu}-k\right) v_{j, \nu}$. The vectors $\left\{X_{i, \ell}\right\}$ and $\left\{Y_{j, \nu}\right\}$ are both linearly independent, and for all $\ell \in J$ and $\nu \in \widetilde{J}$ we have

$$
\left\langle\left[P_{M, \overline{\widetilde{\mathcal{S}}}}\left(A_{B}-\lambda\right)^{-1} P_{N, \overline{\mathcal{S}}}\right]_{\lambda=k} X_{i, \ell}, Y_{j, \nu}\right\rangle=\left\langle\left[\widetilde{P}_{M^{\prime}} M_{B}(\lambda) P_{N^{\prime}}\right]_{\lambda=k} f_{i}, w_{j}\right\rangle .
$$

Define $M_{1}$ to be the matrix with entries

$$
\left\langle\left[P_{M, \overline{\mathcal{S}}}\left(A_{B}-\lambda\right)^{-1} P_{N, \overline{\mathcal{S}}}\right]_{\lambda=k} X_{i, \ell}, Y_{j, \nu}\right\rangle,
$$

ordered by incrementing $\ell$ and $\nu$ before $i$ and $j$, and $M_{2}$ to be the matrix with entries $\left\langle\left[\widetilde{P}_{M^{\prime}} M_{B}(\lambda) P_{N^{\prime}}\right]_{\lambda=k} f_{i}, w_{j}\right\rangle$. It follows from the definition of the Kronecker product that $M_{1}=M_{2} \otimes E$, in which $E$ is the matrix whose entries are all equal to 1 . By consideration of the singular values of the Kronecker product ( $E$ has only one non-zero singular value) it follows that $M_{1}$ and $M_{2}$ have the same rank, and hence that

$$
\operatorname{rank}\left(\left[P_{M, \widetilde{\mathcal{S}}}\left(A_{B}-\lambda\right)^{-1} P_{N, \overline{\mathcal{S}}}\right]_{\lambda=k}\right)=\operatorname{rank}\left(\left[\widetilde{P}_{M^{\prime}} M_{B}(\lambda) P_{N^{\prime}}\right]_{\lambda=k}\right) .
$$

If we define

$$
\operatorname{rank}\left(\left[P_{\overline{\mathcal{S}}}\left(A_{B}-\lambda\right)^{-1} P_{\overline{\mathcal{S}}}\right]_{\lambda=k}\right):=\lim _{M, N \rightarrow \infty} \operatorname{rank}\left(\left[P_{M, \overline{\mathcal{S}}}\left(A_{B}-\lambda\right)^{-1} P_{N, \overline{\mathcal{S}}}\right]_{\lambda=k}\right)
$$

and exploit the fact that the $\left\{f_{i}\right\}_{i \in I}$ and $\left\{v_{j}\right\}_{j \in \tilde{I}}$ exhaust $\mathcal{H}$ and $\mathcal{K}$ respectively, then it follows that

$$
\operatorname{rank}\left(\left[P_{\widetilde{\mathcal{S}}}\left(A_{B}-\lambda\right)^{-1} P_{\overline{\mathcal{S}}}\right]_{\lambda=k}\right)=\operatorname{rank}\left(\left[M_{B}(\lambda)\right]_{\lambda=k}\right) .
$$

7. The detectable subspace for the Friedrichs model. All our results so far have involved important hypotheses about detectable subspaces or about projections or restrictions of resolvents on to detectable subspaces. We have already mentioned the concrete description of these spaces for Schrödinger and Hain-Lüst operators in Section 3 (Proposition 3.1 for the Schrödinger case and the discussion in Subsection 3.2 for HainLüst). We now turn our attention to their description for the seemingly innocuous Friedrichs model. We shall present a very clean characterization of detectable subspaces in terms of an eigenspace of a Hankel-like operator (see Theorem 7.4). This will form the basis of concrete calculations in the second half of this section, including the computation of the defect number 
$\operatorname{def}(\overline{\mathcal{S}}):=\operatorname{dim}\left(\mathcal{S}^{\perp}\right)$ in a particular case. We start with an elementary uniqueness lemma, which is proved in [19]; it also follows from a more general result of Widom [50, pp. 134-135].

Lemma 7.1. Let

$$
G(\mu)= \begin{cases}G_{+}(\mu), & \mu \in \mathbb{C}_{+}, \\ G_{-}(\mu), & \mu \in \mathbb{C}_{-},\end{cases}
$$

with $G_{ \pm} \in H_{1}^{ \pm}+H_{2}^{ \pm}:=\left\{G_{1}+G_{2}: G_{1} \in H_{1}^{ \pm}, G_{2} \in H_{2}^{ \pm}\right\}$. Then the jump across the real axis satisfies $[G] \equiv 0$ if and only if $G \equiv 0$.

We introduce the notation $\hat{x}$ for the Cauchy or Borel transform given by

$$
\widehat{\bar{\phi}}(\lambda)=\left\langle\frac{1}{t-\lambda}, \phi\right\rangle, \quad \widehat{\psi}(\lambda)=\left\langle\frac{1}{t-\lambda}, \bar{\psi}\right\rangle
$$

and $P_{ \pm}: L^{2}(\mathbb{R}) \rightarrow H_{2}^{ \pm}(\mathbb{R})$ for the Riesz projections given by

$$
P_{ \pm} f(k)= \pm \frac{1}{2 \pi i} \lim _{\varepsilon \rightarrow 0} \widehat{f}(k \pm i \varepsilon)= \pm \frac{1}{2 \pi i} \lim _{\varepsilon \rightarrow 0} \int_{\mathbb{R}} \frac{f(x)}{x-(k \pm i \varepsilon)} d x
$$

where the limit is to be understood in $L^{2}(\mathbb{R})$ (see [30]). Here, $H_{p}^{+}(\mathbb{R})$ and $H_{p}^{-}(\mathbb{R})$ denote the Hardy spaces of boundary values of $p$-integrable functions in the upper and lower complex half-plane, respectively. To simplify notation, we also sometimes write $(\widehat{f})_{ \pm}(k)=\widehat{f}(k \pm i 0):=2 \pi i P_{ \pm} f(k)$.

We next give a characterization of the space $\overline{\mathcal{S}}$, or more precisely its orthogonal complement.

Proposition 7.2. Let $P_{ \pm}$be the Riesz projections defined in (7.2). Let $D(\lambda)$ be as in (3.15). Denote by $D_{ \pm}(\lambda)$ its restriction to $\mathbb{C}_{ \pm}$and by $D_{ \pm}$the boundary values of these functions on $\mathbb{R}$ (which exist a.e., cf. [30, 43]).

(1) Let $\phi, \psi \in L^{2}$. Then $g \in \overline{\mathcal{S}}^{\perp}$ if and only if

$$
P_{+} \bar{g}-\frac{2 \pi i}{D_{+}}\left(P_{+} \bar{\phi}\right) P_{+}(\psi \bar{g})=0 \quad \text { and } \quad P_{-} \bar{g}+\frac{2 \pi i}{D_{-}}\left(P_{-} \bar{\phi}\right) P_{-}(\psi \bar{g})=0,
$$

if and only if

$$
\left\{\begin{array}{l}
\text { (i) } \frac{\left(P_{+} \bar{\phi}\right) P_{+}(\psi \bar{g})}{D_{+}} \in H_{2}^{+}, \quad \text { (ii) } \frac{\left(P_{-} \bar{\phi}\right) P_{-}(\psi \bar{g})}{D_{-}} \in H_{2}^{-}, \\
\text {(iii) } \bar{g}-\frac{2 \pi i}{D_{+}}\left(P_{+} \bar{\phi}\right) P_{+}(\psi \bar{g})+\frac{2 \pi i}{D_{-}}\left(P_{-} \bar{\phi}\right) P_{-}(\psi \bar{g})=0 \text { (a.e.). }
\end{array}\right.
$$

(2) If $\phi \in L^{2}$ and $\psi \in L^{2} \cap L^{\infty}$, or $\phi, \psi \in L^{2} \cap L^{4}$, then $g \in \overline{\mathcal{S}}^{\perp}$ if and only if any of the following three equivalent conditions holds:

$$
\begin{aligned}
& {\left[D_{+}-2 \pi i\left(P_{+} \bar{\phi}\right) \psi\right] \bar{g}=2 \pi i \bar{\phi}\left[\psi P_{-} \bar{g}-P_{-}(\psi \bar{g})\right](\text { a.e. }),} \\
& {\left[D_{+}-2 \pi i\left(P_{+} \bar{\phi}\right) \psi\right] \bar{g}=2 \pi i \bar{\phi}\left[-\psi P_{+} \bar{g}+P_{+}(\psi \bar{g})\right](\text { a.e. }),} \\
& {\left[D_{+}-2 \pi i\left(P_{+} \bar{\phi}\right) \psi\right] \bar{g}=2 \pi i \bar{\phi}\left[P_{+}\left(\psi P_{-} \bar{g}\right)-P_{-}\left(\psi P_{+} \bar{g}\right)\right] \text { (a.e.). }}
\end{aligned}
$$


REMARK 7.3. (1) The second part of the proposition allows us to replace all three conditions (i)-(iii) in (7.4) with a single pointwise condition under mild extra assumptions on $\phi$ and/or $\psi$.

(2) Note that the operator $\left[P_{+}\left(\psi P_{-} \bar{g}\right)-P_{-}\left(\psi P_{+} \bar{g}\right)\right]$ in the last characterization of $\overline{\mathcal{S}}^{\perp}$ is the difference of two Hankel operators.

Proof of Proposition 7.2. (i) Noting the form of elements from $\operatorname{Ran} S_{\lambda, B}$ in (3.14), we get

where

$$
\mathcal{S}^{\perp}=\left\{g \in L^{2}(\mathbb{R}): \forall \mu \notin \mathbb{R}, F_{ \pm}(\mu)=0\right\},
$$

$$
F_{ \pm}(\mu):=\left\langle\frac{1}{x-\mu}, g\right\rangle-\frac{1}{D(\mu)}\left\langle\frac{1}{x-\mu}, \phi\right\rangle\left\langle\frac{1}{x-\mu} \psi, g\right\rangle
$$

and the index \pm indicates which half-plane $\mu$ lies in. Then

$$
F_{ \pm}(\mu)=\widehat{\bar{g}}(\mu)-\frac{1}{D(\mu)} \widehat{\bar{\phi}}(\mu) \widehat{\psi \bar{g}}(\mu) \text {. }
$$

First assume $g \in \mathcal{S}^{\perp}$. Let $\mu \in \mathbb{C}_{ \pm} \backslash\{D(\mu)=0\}$. Then $F_{ \pm}(\mu)=0$ is equivalent to

$$
\left(P_{ \pm} \bar{g}\right)(\mu)= \pm \frac{2 \pi i}{D_{ \pm}(\mu)}\left(P_{ \pm} \bar{\phi}\right)(\mu) P_{ \pm}(\psi \bar{g})(\mu) .
$$

This proves the first implication in the statement.

As the zeroes of $D(\mu)$ in $\mathbb{C}_{ \pm}$form a discrete set, the right hand side of (7.9) lies in $H_{2}^{ \pm}$, showing (i) and (ii). Since $\bar{g}=P_{+} \bar{g}+P_{-} \bar{g}$, we also get (iii). For the reverse implications, simply apply $P_{+}$and $P_{-}$to $\bar{g}$ as given in (iii).

(ii) We first show the equivalence in (7.5). Let $g \in \mathcal{S}^{\perp}$ and apply $P_{ \pm}$to part (iii) of (7.4), keeping parts (i) and (ii) in mind. Then

$$
P_{+} \bar{g}-\frac{2 \pi i}{D_{+}}\left(P_{+} \bar{\phi}\right) P_{+}(\psi \bar{g})=0 \quad \text { and } \quad P_{-} \bar{g}+\frac{2 \pi i}{D_{-}}\left(P_{-} \bar{\phi}\right) P_{-}(\psi \bar{g})=0 .
$$

Since $D_{ \pm}$only have discrete zeroes, this is equivalent to

$$
D_{ \pm} P_{ \pm}(\bar{g}) \mp 2 \pi i\left(P_{ \pm} \bar{\phi}\right) P_{ \pm}(\psi \bar{g})=0 .
$$

In particular, on $\mathbb{R}$,

$$
D_{+} P_{+}(\bar{g})-2 \pi i\left(P_{+} \bar{\phi}\right) P_{+}(\psi \bar{g})=-D_{-} P_{-}(\bar{g})-2 \pi i\left(P_{-} \bar{\phi}\right) P_{-}(\psi \bar{g}) \text { (a.e.). }
$$

Since for the boundary values on $\mathbb{R}$ we have $D_{-}=1-2 \pi i P_{-}(\psi \bar{\phi})=D_{+}-$ $2 \pi i \psi \bar{\phi}$ a.e., this is equivalent to

$$
\begin{aligned}
& D_{+}\left(P_{+}(\bar{g})+P_{-}(\bar{g})\right)-2 \pi i\left(P_{+} \bar{\phi}\right) P_{+}(\psi \bar{g}) \\
& \left.\quad=2 \pi i \psi \bar{\phi} P_{-}(\bar{g})-2 \pi i\left(P_{-} \bar{\phi}\right) P_{-}(\psi \bar{g}) \quad \text { (a.e. }\right),
\end{aligned}
$$

which can be rewritten as

$$
\left.D_{+} \bar{g}-2 \pi i\left(P_{+} \bar{\phi}\right) \psi \bar{g}=2 \pi i \psi \bar{\phi} P_{-}(\bar{g})-2 \pi i \bar{\phi} P_{-}(\psi \bar{g}) \quad \text { (a.e. }\right),
$$

giving the right hand side of (7.5). 
On the other hand, assume the right hand side of (7.5) holds. Retracing the steps above gives

$$
\begin{aligned}
\widetilde{F}_{+} & :=D_{+} P_{+}(\bar{g})-2 \pi i\left(P_{+} \bar{\phi}\right) P_{+}(\psi \bar{g}) \\
& =-D_{-} P_{-}(\bar{g})-2 \pi i\left(P_{-} \bar{\phi}\right) P_{-}(\psi \bar{g})=: \widetilde{F}_{-} \quad \text { (a.e.) } .
\end{aligned}
$$

Using Hölder's inequality and boundedness of the Riesz projections $P_{ \pm}$: $L^{p} \rightarrow L^{p}$ for $1<p<\infty$ for the cases $p=4 / 3, p=2$ and $p=4$ (see the proof of Proposition 7.6 for more details), our conditions on $\psi$ and $\phi$ guarantee that $\widetilde{F}_{ \pm} \in H_{1}^{ \pm}+H_{2}^{ \pm}$. Moreover, (7.10) states that $[\widetilde{F}]=0$. By Lemma 7.1 we have $\widetilde{F}=0$ and so

$D_{+} P_{+}(\bar{g})-2 \pi i\left(P_{+} \bar{\phi}\right) P_{+}(\psi \bar{g})=0 \quad$ and $\quad D_{-} P_{-}(\bar{g})+2 \pi i\left(P_{-} \bar{\phi}\right) P_{-}(\psi \bar{g})=0$.

Thus all conditions on the right hand side of (7.3) are satisfied and $g \in \mathcal{S}^{\perp}$.

The identity $P_{-}=I-P_{+}$then gives (7.6), and a similarly simple calculation leads to (7.7).

As an immediate consequence of (7.5), we get

Theorem 7.4. Assume $\phi \in L^{2}$ and $\psi \in L^{2} \cap L^{\infty}$, or $\phi, \psi \in L^{2} \cap L^{4}$, and let $\left({ }^{1}\right)$

$$
\mathcal{D}=\left\{u \in L^{2}(\mathbb{R}):\left[-P_{+}(\psi \bar{\phi})+P_{+}(\bar{\phi}) \psi\right] u+\bar{\phi}\left[\psi P_{-}-P_{-} \psi\right] u \in L^{2}(\mathbb{R})\right\} .
$$

Define the operator $L$ on the domain $D(L):=\mathcal{D}$ by

$$
L u=\left[-P_{+}(\psi \bar{\phi})+P_{+}(\bar{\phi}) \psi\right] u+\bar{\phi}\left[\psi P_{-}-P_{-} \psi\right] u .
$$

Then $\overline{\mathcal{S}} \neq L^{2}(\mathbb{R})$ iff $1 /(2 \pi i) \in \sigma_{p}(L)$ and $\overline{\mathcal{S}}^{\perp}=\operatorname{ker}(L-1 /(2 \pi i))$.

Furthermore, let $\eta \in L^{\infty}(\mathbb{R})$ be a function such that $\eta(k) \neq 0$ a.e. and $\eta\left[-P_{+}(\psi \bar{\phi})+P_{+}(\bar{\phi}) \psi\right], \eta \psi \bar{\phi}, \eta \bar{\phi} \in L^{\infty}(\mathbb{R})$. Define the operator $\mathcal{L}$ in $L^{2}(\mathbb{R})$ by

$$
\mathcal{L} u=\eta\left[-\frac{1}{2 \pi i}-P_{+}(\psi \bar{\phi})+P_{+}(\bar{\phi}) \psi\right] u+\eta \bar{\phi}\left[\psi P_{-}-P_{-} \psi\right] u
$$

with dense domain $\left(^{2}\right) D(\mathcal{L})=\left\{u \in L^{2}(\mathbb{R}): \eta \bar{\phi} P_{-}(\psi u) \in L^{2}(\mathbb{R})\right\}$. Then $\overline{\mathcal{S}} \neq L^{2}(\mathbb{R})$ iff $0 \in \sigma_{p}(\mathcal{L})$. Moreover, $\overline{\mathcal{S}}^{\perp}=\operatorname{ker} \mathcal{L}$.

REMARK 7.5. Replacing $\psi$ by $\alpha \psi$, we denote the corresponding detectable subspace by $\overline{\mathcal{S}}_{\alpha}$. Then, under the conditions in the second part of Proposition 7.2, we get $g \in \mathcal{S}_{\alpha}^{\perp}$ iff

$$
\frac{1}{2 \pi i \alpha} \bar{g}=\left[-P_{+}(\psi \bar{\phi})+P_{+}(\bar{\phi}) \psi\right] \bar{g}+\bar{\phi}\left[P_{+} \psi P_{-}-P_{-} \psi P_{+}\right] \bar{g}=L \bar{g},
$$

$\left({ }^{1}\right)$ Note that $u \in L^{2}(\mathbb{R})$ implies $\left[-P_{+}(\psi \bar{\phi})+P_{+}(\bar{\phi}) \psi\right] u+\bar{\phi}\left[\psi P_{-}-P_{-} \psi\right] u \in L^{1}(\mathbb{R})$.

$\left({ }^{2}\right)$ Note that if $\psi \in L^{\infty}$, then $D(\mathcal{L})=L^{2}(\mathbb{R})$. 
where $L$ is the sum of a multiplication operator and the difference of two Hankel operators multiplied by $\bar{\phi}$. As in the theorem, we get $\mathcal{S}_{\alpha}^{\perp} \neq\{0\}$ iff $1 /(2 \pi i \alpha) \in \sigma_{p}(L)$ and $\mathcal{S}_{\alpha}^{\perp}$ is given by the corresponding kernel.

We now consider several special cases that illustrate the different situations that can arise depending on the support of $\phi$ and $\psi$.

7.1. The case of disjoint supports. In this part we assume that $\phi \psi=0$ almost everywhere, in particular $D(\lambda) \equiv 1$, and that either

$$
\phi \in L^{2}(\mathbb{R}) \text { and } \psi \in L^{2} \cap L^{\infty}, \quad \text { or } \quad \phi, \psi \in L^{2} \cap L^{4} .
$$

In some cases (which will be mentioned later), we will require the slightly stronger condition

$$
\phi \in L^{2}(\mathbb{R}) \cap L^{2+\varepsilon} \text { for some } \varepsilon>0 \text { and } \psi \in L^{2} \cap L^{\infty} \quad \text { or } \quad \phi, \psi \in L^{2} \cap L^{4} \text {. }
$$

Proposition 7.6. Let either $\phi \in L^{2}(\mathbb{R})$ and $\psi \in L^{2} \cap L^{\infty}$, or $\phi, \psi \in$ $L^{2} \cap L^{4}$, be such that $\phi \psi=0$. Then

$$
\begin{aligned}
g \in \overline{\mathcal{S}}^{\perp} & \Leftrightarrow \bar{g}-2 \pi i\left(P_{+} \bar{\phi}\right) P_{+}(\psi \bar{g})+2 \pi i\left(P_{-} \bar{\phi}\right) P_{-}(\psi \bar{g})=0 \text { (a.e.) }, \\
& \Leftrightarrow \bar{g}-2 \pi i \bar{\phi} P_{+}(\psi \bar{g})+2 \pi i\left(P_{-} \bar{\phi}\right) \psi \bar{g}=0 \text { (a.e.) }
\end{aligned}
$$

Define $L_{0}=2 \pi i\left(\bar{\phi} P_{+} \psi-\left(P_{-} \bar{\phi}\right) \psi\right) \equiv 2 \pi i\left(\bar{\phi} P_{+} \psi+\left(P_{+} \bar{\phi}\right) \psi\right)$ on its maximal domain $D\left(L_{0}\right)=\left\{u \in L^{2}(\mathbb{R}):\left(\bar{\phi} P_{+} \psi+\left(P_{+} \bar{\phi}\right) \psi\right) u \in L^{2}(\mathbb{R})\right\}$. Then we have $\overline{\mathcal{S}}=L^{2}(\mathbb{R})$ if and only if 1 is not an eigenvalue of $L_{0}$.

Proof. In this case, $D(\lambda) \equiv 1$, so (7.15) is equivalent to condition (iii) in (7.4) and one implication is trivial. By Proposition 7.2 it is sufficient to show that conditions (i) and (ii) in (7.4) hold. In the present case, they simplify to

$$
\left(\mathrm{i}^{\prime}\right)\left(P_{+} \bar{\phi}\right) P_{+}(\psi \bar{g}) \in H_{2}^{+} \quad \text { and } \quad\left(\mathrm{ii}^{\prime}\right)\left(P_{-} \bar{\phi}\right) P_{-}(\psi \bar{g}) \in H_{2}^{-} \text {. }
$$

When $\phi \in L^{2}(\mathbb{R})$ and $\psi \in L^{2} \cap L^{\infty}$, we have $P_{+} \bar{\phi} \in L^{2}(\mathbb{R})$ and $P_{+}(\psi \bar{g}) \in$ $L^{2}(\mathbb{R})$ by boundedness of the Riesz projection $P_{+}: L^{2}(\mathbb{R}) \rightarrow L^{2}(\mathbb{R})$, so the product lies in $H_{1}^{+}$(see [30]). On the other hand, if $\phi, \psi \in L^{2} \cap L^{4}$, then $\psi \bar{g} \in L^{4 / 3}$ by Hölder's inequality, so $P_{+}(\psi \bar{g}) \in L^{4 / 3}$. Also $P_{+} \bar{\phi} \in L^{4}$, so by Hölder's inequality again, the product lies in $H_{1}^{+}$. Similarly $\left(P_{-} \bar{\phi}\right) P_{-}(\psi \bar{g})$ is in $H_{1}^{-}$.

From $(7.15), \bar{g}=2 \pi i\left(P_{+} \bar{\phi}\right) P_{+}(\psi \bar{g})-2 \pi i\left(P_{-} \bar{\phi}\right) P_{-}(\psi \bar{g})$, which gives a decomposition of $\bar{g} \in L^{2}(\mathbb{R})$ into its unique $H^{+}$and $H^{-}$components, whence we obtain $\left(\mathrm{i}^{\prime}\right)$ and $\left(\mathrm{ii}^{\prime}\right)$.

Now,

$$
\begin{aligned}
\bar{g} & =2 \pi i\left(P_{+} \bar{\phi}\right) P_{+}(\psi \bar{g})-2 \pi i\left(P_{-} \bar{\phi}\right) P_{-}(\psi \bar{g})=2 \pi i\left[\left(P_{+} \bar{\phi}\right) P_{+} \psi-\left(P_{-} \bar{\phi}\right) P_{-} \psi\right] \bar{g} \\
& =2 \pi i\left[\bar{\phi} P_{+} \psi-\left(P_{-} \bar{\phi}\right)\left(P_{+} \psi+P_{-} \psi\right)\right] \bar{g}=L_{0} \bar{g},
\end{aligned}
$$

which shows (7.16) and the statement about $L_{0}$. 
Remark 7.7. Note that $D\left(L_{0}\right)$ is dense in $L^{2}(\mathbb{R})$ if $\phi, \psi \in L^{2} \cap L^{4}$ or if $\phi \in L^{2+\varepsilon}$ for some $\varepsilon>0$ and $\psi \in L^{2} \cap L^{\infty}$, as it contains $L^{2} \cap L^{\infty}$.

Theorem 7.8. Define the sets $\left(^{3}\right) \Omega_{\psi}=\{x \in \mathbb{R}: \psi(x) \neq 0\}, \Omega_{\phi}=$ $\{x \in \mathbb{R}: \phi(x) \neq 0\}$ and $\Omega=\mathbb{R} \backslash\left(\Omega_{\psi} \cup \Omega_{\phi}\right)$. Then $g \in \overline{\mathcal{S}}^{\perp}$ iff $g \in L^{2}(\mathbb{R})$ and

(a) $\chi_{\Omega_{\phi}} \bar{g}(k)=\bar{\phi}(k) \widehat{\psi \chi_{\Omega_{\psi}} \bar{g}}(k+i 0)$,

(b) $\chi_{\Omega_{\psi}} \bar{g}(k)(1-\widehat{\bar{\phi}}(k-i 0) \psi(k))=0$,

(c) $\left.g\right|_{\Omega}=0$.

Let $\Omega_{\psi, 0}=\{k \in \mathbb{R}: \widehat{\bar{\phi}}(k-i 0) \psi(k)=1\}$. Then, additionally:

(i) If $\Omega_{\psi, 0}$ has zero measure, then $\overline{\mathcal{S}}=L^{2}(\mathbb{R})$.

(ii) Assume (7.14). If $\Omega_{\psi, 0}$ has non-zero measure, then $\overline{\mathcal{S}}^{\perp} \neq\{0\}$. Moreover,

$$
\overline{\mathcal{S}} \subseteq\left\{f \in L^{2}(\mathbb{R}): f=\psi(\widehat{f \bar{\phi}})_{-} \text {on } \Omega_{\psi, 0}\right\} .
$$

(iii) Assume $\phi, \psi \in L^{2} \cap L^{\infty}$. Then

$$
\overline{\mathcal{S}}=\left\{f \in L^{2}(\mathbb{R}): f=\psi(\widehat{f \bar{\phi}})_{-} \text {on } \Omega_{\psi, 0}\right\} .
$$

REMARK 7.9. (1) Note that (7.17) shows that $\left.g\right|_{\Omega_{\psi}}$ completely determines $\left.g\right|_{\Omega_{\phi}}$.

(2) Condition (7.17) gives an additional restriction on $\left.g\right|_{\Omega_{\psi}}$, requiring that $\bar{\phi}(k) \widehat{\psi \chi_{\Omega_{\psi}} \bar{g}}(k+i 0) \in L^{2}\left(\Omega_{\phi}\right)$.

(3) Condition (7.14) implies (via the Hölder inequality and boundedness of $P_{+}: L^{p} \rightarrow L^{p}$ for $\left.1<p<\infty\right)$ that for $g \in\left(L^{2} \cap L^{\infty}\right)\left(\Omega_{\psi}\right)$ we have $\bar{\phi}(k) \widehat{\psi \chi_{\Omega_{\psi}} \bar{g}}(k+i 0) \in L^{2}\left(\Omega_{\phi}\right)$.

Proof of Theorem 7.8. We recall that $\mathbb{R}=\Omega_{\phi} \cup \Omega_{\psi} \cup \Omega$. Without loss of generality, we may assume $\Omega_{\phi} \cap \Omega_{\psi}=\emptyset$. Suppose $g \in \overline{\mathcal{S}}^{\perp}$. Using (7.16), we then have three cases:

- $k \in \Omega_{\phi}$. Then $0=\bar{g}(k)-\bar{\phi}(k) \widehat{\psi \chi_{\Omega_{\psi}} \bar{g}}(k+i 0)$. Hence, $\chi_{\Omega_{\phi}} \bar{g}(k)=$ $\bar{\phi}(k) \widehat{\psi \chi_{\Omega_{\psi}} \bar{g}}(k+i 0)$ and so $\left.g\right|_{\Omega_{\psi}}$ completely determines $\left.g\right|_{\Omega_{\phi}}$.

- $k \in \Omega_{\psi}$. Then $0=\bar{g}(k)-\widehat{\bar{\phi}}(k-i 0) \psi(k) \bar{g}(k)$. Hence, almost everywhere,

$$
\chi_{\Omega_{\psi}} \bar{g}(k)(1-\widehat{\bar{\phi}}(k-i 0) \psi(k))=0 .
$$

- $k \in \Omega$. Then $\left.g\right|_{\Omega}=0$.

$\left({ }^{3}\right)$ Note that $\Omega_{\psi}, \Omega_{\phi}$ are only defined up to a set of Lebesgue measure zero, but this is sufficient for our purpose. Also, they may be much smaller than the support of the functions. 
This gives three necessary and sufficient conditions for $g$ to lie in $\overline{\mathcal{S}}^{\perp}$. We now prove statements (i)-(iii).

(i) If $\widehat{\bar{\phi}}(k-i 0) \psi(k) \neq 1$ for almost every $k \in \Omega_{\psi},(7.17)(\mathrm{b})$ implies that $\left.g\right|_{\Omega_{\psi}}=0$, and so by (7.17) we have $\left.g\right|_{\Omega_{\phi}}=0$, whence $g \equiv 0$ and $\overline{\mathcal{S}}=L^{2}(\mathbb{R})$.

(ii) Choose $g$ on $\Omega_{\psi, 0}$ to be an arbitrary non-zero $\left(L^{2} \cap L^{\infty}\right)\left(\Omega_{\psi, 0}\right)$ function (in the case when $\phi \in L^{2+\varepsilon}, \psi \in L^{\infty}$, we may even choose $g$ arbitrary in $\left.\left(L^{2} \cap L^{2(2+\varepsilon) / \varepsilon}\right)\left(\Omega_{\psi, 0}\right)\right)$. Extending $g$ by zero to $\Omega_{\psi}$ and then using Hölder's inequality and boundedness of $P_{+}$, we automatically get $\bar{\phi}\left(\widehat{\psi \chi_{\Omega_{\psi}}} \bar{g}\right)_{+} \in L^{2}(\mathbb{R})$. This then determines $g$ on $\Omega_{\psi}$ from $(7.17)$, and extending $g$ to $\Omega$ by 0 we have $g \in \mathcal{S}^{\perp}$.

Now let $f \in \overline{\mathcal{S}}$ and choose $g \in\left(L^{2} \cap L^{\infty}\right)\left(\Omega_{\psi, 0}\right)$. Then

$$
\begin{aligned}
0 & =\int_{\mathbb{R}} f \bar{g}=\int_{\Omega_{\psi, 0}} f \bar{g}+\int_{\Omega_{\phi}} f \bar{g}=\int_{\Omega_{\psi, 0}} f \bar{g}+\int_{\Omega_{\phi}} f \bar{\phi}(\widehat{\psi \bar{g}})_{+} \\
& =\int_{\Omega_{\psi, 0}} f \bar{g}+\int_{\mathbb{R}} f \bar{\phi}(\widehat{\psi \bar{g}})_{+}=\int_{\Omega_{\psi, 0}} f \bar{g}-\int_{\mathbb{R}}(\widehat{f \bar{\phi}})_{-} \psi \bar{g} \\
& =\int_{\Omega_{\psi, 0}} f \bar{g}-\int_{\Omega_{\psi, 0}}(\widehat{f \bar{\phi}})_{-} \psi \bar{g}=\int_{\Omega_{\psi, 0}}\left(f-\psi(\widehat{f \bar{\phi}})_{-}\right) \bar{g},
\end{aligned}
$$

from which it follows that $\bar{g} \mapsto \int_{\Omega_{\psi, 0}}\left(f-\psi(\widehat{f \bar{\phi}})_{-}\right) \bar{g}$ is a bounded linear functional for all $g$ in the dense set $\left(L^{2} \cap L^{\infty}\right)\left(\Omega_{\psi, 0}\right)$. Hence, $\psi(\widehat{f \bar{\phi}})_{-}$is in $L^{2}\left(\Omega_{\psi, 0}\right)$, and we see that $f \in \overline{\mathcal{S}}$ must satisfy $f=\psi(\widehat{f \bar{\phi}})_{-}$on $L^{2}\left(\Omega_{\psi, 0}\right)$.

(iii) If $\psi$ and $\phi$ are both bounded, then choosing $g \in L^{2} \cap L^{\infty}\left(\Omega_{\psi, 0}\right)$ and determining $g$ on $\Omega_{\phi}$ from (7.17) will give a dense set of $g$ in $\mathcal{S}^{\perp}$, as $g \mapsto \bar{\phi}(\widehat{\psi} \bar{g})_{+}$is bounded. The calculation in (ii) can then be repeated for a dense set of $g$ in $\mathcal{S}^{\perp}$, which gives the needed equality.

EXAmple 7.10. Let $I$ and $I^{\prime}$ be disjoint closed intervals in $\mathbb{R}$. Choose $\phi \in L^{\infty}$ with $\operatorname{supp} \phi \in I$ such that

$$
\int_{\mathbb{R}} \frac{\overline{\phi(x)}}{x-k} d x \neq 0 \quad \text { for } k \in I^{\prime}
$$

Define

$$
\psi(k)= \begin{cases}\left(\int_{\mathbb{R}} \frac{\overline{\phi(x)}}{x-k} d x\right)^{-1} & \text { for } k \in I^{\prime}, \\ 0 & \text { otherwise. }\end{cases}
$$

Then $\psi \in L^{\infty}, \phi \psi=0$ and $\widehat{\bar{\phi}}(k-i 0) \psi(k)=1$. Therefore, by Theorem 7.8(ii), $\overline{\mathcal{S}}^{\perp} \neq\{0\}$. 
Theorem 7.11. Assume (7.14). Then $\overline{\mathcal{S}}=L^{2}(\mathbb{R}) \Leftrightarrow 1-\psi(\widehat{\bar{\phi}})(k+i 0) \neq 0$ for a.e. $k \in \mathbb{R}$. Moreover,

$$
\operatorname{def}(\mathcal{S})=\operatorname{dim}\left(\mathcal{S}^{\perp}\right)= \begin{cases}0 & \text { if } \overline{\mathcal{S}}=L^{2}(\mathbb{R}), \\ \infty & \text { otherwise }\end{cases}
$$

Proof. From (7.16), we have

$$
\begin{aligned}
g \in \mathcal{S}^{\perp} & \Leftrightarrow \bar{g}(k)-\bar{\phi}(k)(\widehat{\psi \bar{g}})_{+}-(\widehat{\bar{\phi}})_{-} \psi \bar{g}=0 \text { a.e. } \\
& \Leftrightarrow\left(1-\psi(\widehat{\bar{\phi}})_{+}\right)\left(\bar{g}-\bar{\phi}(\widehat{\psi \bar{g}})_{+}\right)=0 \text { a.e. }
\end{aligned}
$$

We have two cases: In the first, $\bar{g}-\bar{\phi}(\widehat{\psi} \bar{g})_{+}=0$ a.e. Then multiplying by $\psi$ and using the condition on the supports, we get $\psi \bar{g}=0$ and hence $\bar{g}=0$.

In the second case, $\bar{g}-\bar{\phi} \cdot 2 \pi i P_{+}(\psi \bar{g}) \not \equiv 0$. Then there exists a set $E$ of positive measure such that $\bar{g}-\bar{\phi} \cdot 2 \pi i P_{+}(\psi \bar{g}) \neq 0$ on $E$ and $\left.(1-\psi(\widehat{\bar{\phi}}))\right|_{E}=0$ a.e.

We now show that if there exists a set $E$ of positive measure such that $\left.\left(1-\psi(\widehat{\bar{\phi}})_{+}\right)\right|_{E}=0$ a.e., then $\mathcal{S}^{\perp} \neq\{0\}$. Note first that $E \subset \Omega_{\psi}$. Choose any non-zero $\tilde{g} \in\left(L^{2} \cap L^{\infty}\right)(E), \tilde{g} \neq 0$ and continue it to $\mathbb{R}$ by zero. Define

$$
\bar{g}(k)= \begin{cases}\overline{\tilde{g}(k)}, & k \in E, \\ 0, & k \in \Omega_{\psi} \backslash E, \\ \bar{\phi} 2 \pi i P_{+}(\psi \overline{\tilde{g}}), & k \notin \Omega_{\psi} .\end{cases}
$$

By (7.19), to show $g \in \mathcal{S}^{\perp}$ we only require $g \in L^{2}$. This follows immediately from the condition (7.14). From the freedom in the choice of $\tilde{g}$, it is clear that $\operatorname{def}(\mathcal{S})$ is infinite.

To obtain the following results on complete detectability, i.e. $\overline{\mathcal{S}}=L^{2}(\mathbb{R})$, we first prove a lemma.

Lemma 7.12. Let $f: \mathbb{R} \rightarrow \mathbb{C}$ be measurable. For each $\alpha \in \mathbb{C}$, let $E_{\alpha}:=$ $\{x \in \mathbb{R}: f(x)=\alpha\}$. Then the set $\left\{\alpha \in \mathbb{C}:\left|E_{\alpha}\right|>0\right\}$ is countable.

Proof. Notice that $\left\{\alpha \in \mathbb{C}:\left|E_{\alpha}\right|>0\right\}=\bigcup_{n=1}^{\infty}\left\{\alpha \in \mathbb{C}:\left|E_{\alpha}\right|>\right.$ $1 / n\}$. Assume this set is uncountable. Then there exists $\varepsilon_{0}$ such that $J:=$ $\left\{\alpha \in \mathbb{C}:\left|E_{\alpha}\right|>\varepsilon_{0}\right\}$ is uncountable. Moreover, if $\alpha \neq \alpha^{\prime}$, then $E_{\alpha} \cap E_{\alpha^{\prime}}=\emptyset$. Now $\mathbb{C} \supset \bigcup_{\alpha \in J} E_{\alpha}$ is a disjoint union of uncountably many sets of measure greater than $\varepsilon_{0}$. Choose $\widetilde{f}_{\alpha}=\chi_{\widetilde{E}_{\alpha}}$ where $\widetilde{E}_{\alpha} \subseteq E_{\alpha}$ with $\left|\widetilde{E}_{\alpha}\right|=\varepsilon_{0}$. Then $\left\|\widetilde{f}_{\alpha}\right\|=\sqrt{\varepsilon_{0}}$ and $\widetilde{f}_{\alpha} \perp \widetilde{f}_{\alpha^{\prime}}$ for $\alpha \neq \alpha^{\prime}$, contradicting separability of $L^{2}\left(\mathbb{R}^{2}\right)$; therefore $\left\{\alpha \in \mathbb{C}:\left|E_{\alpha}\right|>0\right\}$ is countable.

THEOREM 7.13.

(1) Complete detectability is generic in the following sense. Replace $\psi$ by $\alpha \psi$ for $\alpha \in \mathbb{C}($ or $\phi$ by $\bar{\alpha} \phi)$; then for all $\alpha$ outside a countable set $E_{0}$ we have $\overline{\mathcal{S}}_{\alpha}=L^{2}(\mathbb{R})$, where $\overline{\mathcal{S}}_{\alpha}$ is as defined in Remark 7.5. 
(2) For small perturbations, if $\psi \in L^{\infty}$ and $P_{+} \phi$ or $P_{-} \phi \in L^{\infty}$, replacing $\psi$ by $\alpha \psi$ where $\alpha \in \mathbb{C}$, we get $\overline{\mathcal{S}}_{\alpha}=L^{2}(\mathbb{R})$ for sufficiently small $|\alpha|$.

Proof. (1) This is immediate from Theorem 7.11 with the help of Lemma 7.12 .

(2) From (7.16), we have $g \in \mathcal{S}^{\perp}$ if and only if

$$
\bar{g}(k)-\bar{\phi}(k) \widehat{\psi \bar{g}}(k+i 0)-\widehat{\bar{\phi}}(k-i 0) \psi(k) \bar{g}(k)=0 .
$$

Multiplying by $\psi(k)$, and setting $g_{\psi}:=\psi \bar{g}$, we get

$$
g_{\psi}(k)=\psi(k) \widehat{\bar{\phi}}(k-i 0) g_{\psi}(k) .
$$

Hence, unless $\psi(k) \widehat{\bar{\phi}}(k-i 0)=1$ on a set of non-zero measure, we have $g_{\psi} \equiv 0$, which by $(7.20)$ gives $g \equiv 0$, and therefore $\overline{\mathcal{S}}=L^{2}(\mathbb{R})$. Now, as $\bar{\phi} \psi=0$,

$$
\alpha \psi(k) \widehat{\bar{\phi}}(k-i 0)=-2 \pi i \alpha \psi(k) P_{-} \bar{\phi}=2 \pi i \alpha \psi(k) P_{+} \bar{\phi} .
$$

Assuming $\psi \in L^{\infty}$ and $P_{+} \phi$ or $P_{-} \bar{\phi}$ in $L^{\infty}$, we see that $\overline{\mathcal{S}}_{\alpha}=L^{2}(\mathbb{R})$ for sufficiently small $|\alpha|$, since then $|\alpha \psi(k) \widehat{\bar{\phi}}(k-i 0)|<1$ and (7.21) implies $g_{\psi} \equiv 0$.

We next give a specific example with disjoint supports which illustrates some of the abstract results. The lengthy calculations can be found in [19].

REMARK 7.14. Suppose that $I$ and $I^{\prime}$ are disjoint closed intervals such that $I^{\prime}$ lies to the left of $I$. Let

$$
\phi=\chi_{I} \quad \text { and } \quad \psi(x)=\chi_{I^{\prime}}(x) \cdot\left(\int_{I} \frac{d t}{t-x}\right)^{-1} .
$$

By (quite extensive) explicit calculations, the following may be proved:

(1) In this case $\overline{\mathcal{S}} \neq L^{2}(\mathbb{R})$ with $\operatorname{def}(\overline{\mathcal{S}})=\infty$, while $\overline{\widetilde{\mathcal{S}}}=L^{2}(\mathbb{R})$.

(2) The jump of the $M$-function across the real axis at $k$ is given by

$$
\left[M_{B}^{-1}(k)\right]= \begin{cases}2 \pi i, & k \in \mathbb{R} \backslash\left(I \cup I^{\prime}\right), \\ 2 \pi i(1-\widehat{\psi}(k)), & k \in I, \\ 0, & k \in I^{\prime} .\end{cases}
$$

Moreover, the bordered resolvent $P_{\widetilde{\mathcal{S}}}\left(A_{B}-\lambda\right)^{-1} P_{\overline{\mathcal{S}}}$ jumps at $k \in \mathbb{R}$ iff $k \notin I^{\prime}$, i.e. the location of the jumps of the bordered resolvent coincides with the jumps of $M_{B}$. (Compare to Theorem 6.5 , where we can only border the resovent by finite-dimensional projections.)

7.2. Results when $\phi$ and $\psi$ are not disjointly supported. We present some initial results for the more general case when $\phi$ and $\psi$ do not necessarily have disjoint supports. We plan to address this question in more detail in a forthcoming paper. 
THEOREM 7.15.

(1) Let $\Omega^{c}=\{x: \phi(x) \neq 0\} \cup\{x: \psi(x) \neq 0\}$. Then $g \in \mathcal{S}^{\perp}$ implies $\{x: g(x) \neq 0\} \subseteq \Omega^{c}$ (up to a set of measure zero). In particular, $\mathcal{S}^{\perp} \subseteq L^{2}\left(\Omega^{c}\right)$ and $\overline{\mathcal{S}} \supseteq L^{2}(\Omega)$.

(2) Consider $\psi=\alpha \chi_{I}$ for some constant $\alpha$ and a set I of finite measure, and assume $\left.\phi\right|_{I^{c}}=0$ a.e. Then $\overline{\mathcal{S}}=L^{2}(\mathbb{R})$.

Proof. (1) Let

$$
F_{ \pm}:=P_{ \pm} \bar{g} \mp \frac{2 \pi i}{D_{ \pm}}\left(P_{ \pm} \bar{\phi}\right) P_{ \pm}(\psi \bar{g})=0 .
$$

We consider the condition (7.3). Then $g \in \mathcal{S}^{\perp}$ implies $F_{ \pm} \equiv 0$ and $[F]=0$. On $\Omega$ we see that

$$
\left\langle\frac{1}{x-\mu}, \phi\right\rangle\left\langle\frac{1}{x-\mu} \psi, g\right\rangle\left(1+\left\langle\frac{\psi}{x-\mu}, \phi\right\rangle\right)^{-1}
$$

is analytic a.e., so its jump is zero. Therefore

$$
2 \pi i g(k)=\left.\left\langle\frac{1}{x-\mu}, g\right\rangle\right|_{\Omega}=0 \quad \text { a.e. }
$$

and $g$ vanishes a.e. on $\Omega$. Since our conditions are symmetric in $\phi$ and $\psi$, we immediately also get $\widetilde{\mathcal{S}}^{\perp} \subseteq L^{2}\left(\Omega^{c}\right)$.

(2) Consider $F_{ \pm}$from (7.22). We need to show that if $F$ vanishes, then so does $g$. We have

$$
D_{ \pm}(\mu)=1+\alpha \int_{I} \frac{\bar{\phi}}{x-\mu} d x
$$

so

$$
F_{ \pm}(\mu)=\left\langle\frac{1}{x-\mu}, g\right\rangle-\frac{\alpha \int_{I} \frac{\bar{\phi}}{x-\mu} d x \int_{I} \frac{\bar{g}}{x-\mu} d x}{1+\alpha \int_{I} \frac{\bar{\phi}}{x-\mu} d x} .
$$

Since $g \in \mathcal{S}^{\perp}$, the first part of the theorem implies $\left.g\right|_{I^{c}}=0$ a.e., so

$$
F_{ \pm}(\mu)=\int_{I} \frac{\bar{g}}{x-\mu} d x \frac{1}{1+\alpha \int_{I} \frac{\bar{\phi}}{x-\mu} d x} .
$$

Clearly, $\left(1+\alpha \int_{I} \frac{\bar{\phi}}{x-\mu} d x\right)^{-1} \neq 0$ for a.e. $\mu$, so for all $\mu \notin \mathbb{R}$ we have

$$
\int_{I} \frac{\bar{g}}{x-\mu} d x=\left\langle\frac{1}{x-\mu}, g\right\rangle=0 .
$$

As in the proof of Lemma 7.1, this implies $g \equiv 0$.

We finish this subsection by showing that in our situation we can improve on Theorems 4.1 and 4.6 by recovering the $M$-function from one bordered resolvent. 
TheOREM 7.16. As before, let $\Omega_{\psi}=\{x \in \mathbb{R}: \psi(x) \neq 0\}$ and $\Omega_{\phi}=\{x \in \mathbb{R}:$ $\phi(x) \neq 0\}$. Let $\Omega=\mathbb{R} \backslash\left(\Omega_{\phi} \cup \Omega_{\psi}\right)$ and assume we know a set of non-zero measure $\Omega^{\prime} \subseteq \Omega$. Then $M_{B}(\lambda)$ can be recovered from one bordered resolvent.

REMARK 7.17. The converse is not possible. The asymptotics of the $M$ function at $i \infty$ allows us to recover $B$ and thus $\widehat{\psi}(\lambda) \widehat{\bar{\phi}}(\lambda)$ for any $\lambda$. However, only knowing the product makes it impossible, for example, to distinguish the expression for $A$ from the operator expression obtained by replacing $\psi$ by $\bar{\phi}$ and $\phi$ by $\bar{\psi}$, respectively.

Proof of Theorem 7.16. From Theorem 7.15, we know that $L^{2}\left(\Omega^{\prime}\right) \subseteq$ $\overline{\mathcal{S}} \cap \overline{\widetilde{\mathcal{S}}}$. Choose $v, \widetilde{v} \in L^{2}\left(\Omega^{\prime}\right)$. By assumption, we know $\left\langle\left(A_{B}-\lambda\right)^{-1} v, \widetilde{v}\right\rangle$. Noting that $v \phi=0$ and $\widetilde{v} \psi=0$, from (3.17) and (3.18) we get

$$
\left\langle\left(A_{B}-\lambda\right)^{-1} v, \widetilde{v}\right\rangle-\left\langle\frac{v}{x-\lambda}, \widetilde{v}\right\rangle=-M_{B}(\lambda)\left\langle\frac{v}{x-\lambda}, \mathbf{1}\right\rangle\left\langle\frac{1}{x-\lambda}, \widetilde{v}\right\rangle .
$$

Choosing $v, \widetilde{v} \geq 0$ and not identically zero, we can divide $\lambda$-a.e. to obtain

$$
M_{B}(\lambda)=\frac{\left\langle\frac{v}{x-\lambda}, \widetilde{v}\right\rangle-\left\langle\left(A_{B}-\lambda\right)^{-1} v, \widetilde{v}\right\rangle}{\left\langle\frac{v}{x-\lambda}, \mathbf{1}\right\rangle\left\langle\frac{1}{x-\lambda}, \widetilde{v}\right\rangle} .
$$

Acknowledgments. M. Marletta and S. N. Naboko gratefully acknowledge the support of the Leverhulme Trust, grant RPG167, and of the Wales Institute of Mathematical and Computational Sciences. S. N. Naboko was also partially supported by the Russian Science Foundation (project no. 1511-30007) and the EU Marie Curie grant PIIF-GA-2011-299919.

\section{References}

[1] H. Abels, G. Grubb and I. G. Wood, Extension theory and Krein-type resolvent formulas for nonsmooth boundary value problems, J. Funct. Anal. 266 (2014), 40374100.

[2] D. Alpay and J. Behrndt, Generalized Q-functions and Dirichlet-to-Neumann maps for elliptic differential operators, J. Funct. Anal. 257 (2009), 1666-1694.

[3] W. O. Amrein and D. B. Pearson, $M$ operators: a generalization of Weyl-Titchmarsh theory, J. Comput. Appl. Math. 171 (2004), 1-26.

[4] Yu. Arlinskiur, S. Hassi and H. de Snoo, Q-functions of quasi-selfadjoint contractions, in: Oper. Theory Adv. Appl. 163, Birkhäuser, 2005, 23-54.

[5] Yu. Arlinskiǔ and L. Klotz, Weyl functions of bounded quasi-selfadjoint operators and block operator Jacobi matrices, Acta Sci. Math. (Szeged) 76 (2010), 585-626.

[6] D. Z. Arov and M. A. Nudel'man, Tests for the similarity of all minimal passive realizations of a fixed transfer function (scattering or resistance matrix), Sb. Math. 193 (2002), 791-810.

[7] J. Behrndt and M. Langer, Boundary value problems for elliptic partial differential operators on bounded domains, J. Funct. Anal. 243 (2007), 536-565. 
[8] J. Behrndt and M. Langer, Elliptic operators, Dirichlet-to-Neumann maps and quasi-boundary triples, in: London Math. Soc. Lecture Note Ser. 404, Cambridge Univ. Press, Cambridge, 2012, 121-160.

[9] J. Behrndt, M. Langer and V. Lotoreichik, Trace formulae and singular values of resolvent power differences of self-adjoint elliptic operators, J. London Math. Soc. 88 (2013), 319-337.

[10] J. Behrndt, M. Langer and V. Lotoreichik, Spectral estimates for resolvent differences of self-adjoint elliptic operators, Integral Equations Operator Theory 77 (2013), 1-37.

[11] J. Behrndt and T. Micheler, Elliptic differential operators on Lipschitz domains and abstract boundary value problems, J. Funct. Anal. 267 (2014), 3657-3709.

[12] J. Behrndt and J. Rohleder, An inverse problem of Calderón type with partial data, Comm. Partial Differential Equations 37 (2012), 1141-1159.

[13] G. Borg, Uniqueness theorems in the spectral theory of $y^{\prime \prime}+(\lambda-q(x)) y=0$, in: Den 11te Skandinaviske Matematikerkongress (Trondheim, 1949), Johan Grundt Tanums Forlag, Oslo, 1952, 276-287.

[14] M. S. Brodskiǔ, Triangular and Jordan Representations of Linear Operators, Transl. Math. Monogr. 32, Amer. Math. Soc., Providence, RI, 1971.

[15] B. M. Brown, G. Grubb and I. G. Wood, $M$-functions for closed extensions of adjoint pairs of operators with applications to elliptic boundary problems, Math. Nachr. 282 (2009), 314-347.

[16] M. Brown, J. Hinchcliffe, M. Marletta, S. Naboko and I. Wood, The abstract Titchmarsh-Weyl $M$-function for adjoint operator pairs and its relation to the spectrum, Integral Equations Operator Theory 63 (2009), 297-320.

[17] M. Brown, M. Marletta, S. Naboko and I. Wood, Boundary triplets and $M$-functions for non-selfadjoint operators, with applications to elliptic PDEs and block operator matrices, J. London Math. Soc. (2) 77 (2008), 700-718.

[18] B. M. Brown, M. Marletta, S. N. Naboko and I. G. Wood, Detectable subspaces and inverse problems for Hain-Lüst-type operators, Math. Nachr. 289 (2016), 2108-2132.

[19] B. M. Brown, M. Marletta, S. Naboko and I. Wood, An abstract inverse problem for boundary triples with an application to the Friedrichs Model, arXiv:1404.6820v2 (2014).

[20] V. Derkach, S. Hassi, M. Malamud, and H. de Snoo, Boundary relations and generalized resolvents of symmetric operators, Russ. J. Math. Phys. 16 (2009), 17-60.

[21] V. Derkach and M. Malamud, Generalized resolvents and the boundary value problems for Hermitian operators with gaps, J. Funct. Anal. 95 (1991), 1-95.

[22] F. Gesztesy and M. Mitrea, Nonlocal Robin Laplacians and some remarks on a paper by Filonov on eigenvalue inequalities, J. Differential Equations 247 (2009), 2871-2896.

[23] F. Gesztesy and M. Mitrea, Robin-to-Robin maps and Krein-type resolvent formulas for Schrödinger operators on bounded Lipschitz domains, in: Oper. Theory Adv. Appl. 191, Birkhäuser, Basel, 2009, 81-113.

[24] F. Gesztesy and M. Mitrea, A description of all self-adjoint extensions of the Laplacian and Krein-type resolvent formulas on non-smooth domains, J. Anal. Math. 113 (2011), 53-172.

[25] F. Gesztesy, M. Mitrea and M. Zinchenko, Variations on a theme by Jost and Pais, J. Funct. Anal. 253 (2007), 399-448.

[26] F. Gesztesy, S. N. Naboko, R. Weikard and M. Zinchenko, Donoghue-type m-functions for Schrödinger operators with operator-valued potentials, J. Anal. Math., to appear; arXiv:1506.06324 (2015). 
[27] G. Grubb, Krein resolvent formulas for elliptic boundary problems in nonsmooth domains, Rend. Sem. Mat. Univ. Politec. Torino 66 (2008), 271-297.

[28] S. Hassi, M. Malamud and V. Mogilevskii, Unitary equivalence of proper extensions of a symmetric operator and the Weyl function, Integral Equations Operator Theory 77 (2013), 449-487.

[29] V. Isakov, Inverse Problems for Partial Differential Equations, Appl. Math. Sci. 127, Springer, New York, 1998.

[30] P. Koosis, Introduction to $H_{p}$ Spaces, 2nd ed., Cambridge Tracts in Math. 115, Cambridge Univ. Press, Cambridge, 1998.

[31] P. Koosis, The Logarithmic Integral, Vol. I, Cambridge Tracts in Math. 12, Cambridge Univ. Press, Cambridge, 1998.

[32] N. D. Kopachevskiı̆ and S. G. Kreı̆n, An abstract Green formula for a triple of Hilbert spaces, and abstract boundary value and spectral problems, Ukr. Math. Bull. 1 (2004), 77-105.

[33] M. G. Kreı̆n und H. Langer, Über die Q-Funktion eines $\pi$-hermiteschen Operators im Raume $\Pi_{\kappa}$, Acta Sci. Math. (Szeged) 34 (1973), 191-230.

[34] H. Langer and B. Textorius, On generalized resolvents and Q-functions of symmetric linear relations (subspaces) in Hilbert space, Pacific J. Math. 72 (1977), 135-165.

[35] V. E. Lyantse and O. G. Storozh, Methods of the Theory of Unbounded Operators, Naukova Dumka, Kiev, 1983 (in Russian).

[36] M. M. Malamud, Spectral theory of elliptic operators in exterior domains, Russ. J. Math. Phys. 17 (2010), 96-125.

[37] M. M. Malamud and V. M. Mogilevskii, On Weyl functions and Q-functions of dual pairs of linear relations, Dopov. Nats. Akad. Nauk Ukr. Mat. Prirodozn. Tekh. Nauki 1999, no. 4, 32-37.

[38] M. M. Malamud and V. M. Mogilevskii, Krę̆n type formula for canonical resolvents of dual pairs of linear relations, Methods Funct. Anal. Topology 8 (2002), no. 4, $72-100$.

[39] V. A. Marchenko, Concerning the theory of a differential operator of the second order, Dokl. Akad. Nauk SSSR (N.S.) 72 (1950), 457-460 (in Russian).

[40] V. A. Mikhailets and A. V. Sobolev, Common eigenvalue problem and periodic Schrödinger operators, J. Funct. Anal. 165 (1999), 150-172.

[41] A. Posilicano, Self-adjoint extensions of restrictions, Oper. Matrices 2 (2008), 483506.

[42] A. Posilicano and L. Raimondi, Krein's resolvent formula for self-adjoint extensions of symmetric second-order elliptic differential operators, J. Phys. A Math. Theor. 42 (2009), no. 1, 015204, 11 pp.

[43] I. I. Privalov, Boundary Properties of Analytic Functions, 2nd ed. Gos. Izdat. Tekhn.-Teor. Lit., Moscow, 1950 (in Russian).

[44] V. Ryzhov, A general boundary value problem and its Weyl function, Opuscula Math. 27 (2007), 305-331.

[45] V. Ryzhov, Weyl-Titchmarsh function of an abstract boundary value problem, operator colligations, and linear systems with boundary control, Complex Anal. Oper. Theory 3 (2009), 289-322.

[46] O. Staffans, Well-Posed Linear Systems, Encyclopedia Math. Appl. 103, Cambridge Univ. Press, Cambridge, 2005.

[47] J. Sylvester and G. Uhlmann, A uniqueness theorem for an inverse boundary value problem in electrical prospection, Comm. Pure Appl. Math. 39 (1986), 91-112.

[48] J. Sylvester and G. Uhlmann, A global uniqueness theorem for an inverse boundary value problem, Ann. of Math. (2) 125 (1987), 153-169. 
[49] B. Sz.-Nagy and C. Foiaş, Harmonic Analysis of Operators on Hilbert Space, NorthHolland, Amsterdam, 1970.

[50] H. Widom, Singular integral equations in $L_{p}$, Trans. Amer. Math. Soc. 97 (1960), 131-160.

B. M. Brown

Cardiff School of Computer Science and Informatics

Cardiff University

Queen's Buildings

5 The Parade

Cardiff CF24 3AA, UK

E-mail: Malcolm.Brown@cs.cardiff.ac.uk

S. Naboko

Department of Mathematical Physics

Institute of Physics

St. Petersburg State University

1 Ulianovskaia, St. Petergoff

St. Petersburg, 198504, Russia

E-mail: sergey.naboko@gmail.com
M. Marletta

School of Mathematics

Cardiff University

Senghennydd Road

Cardiff CF24 4AG, UK

E-mail: MarlettaM@cardiff.ac.uk

I. Wood

School of Mathematics, Statistics and Actuarial Science University of Kent Cornwallis Building Canterbury CT2 7NF, UK E-mail: i.wood@kent.ac.uk 